\title{
A Highly Crystalline Anthracene-Based MOF-74 Series Featuring Electrical Conductivity and Luminescence
}

Patricia Scheurle, Andre Mähringer, Andreas Jakowetz, Pouya Hosseini, Alexander Richter, Gunther Wittstock, Dana D. Medina, Thomas Bein

Submitted date: 04/06/2019 - Posted date: 05/06/2019

Licence: CC BY-NC-ND 4.0

Citation information: Scheurle, Patricia; Mähringer, Andre; Jakowetz, Andreas; Hosseini, Pouya; Richter, Alexander; Wittstock, Gunther; et al. (2019): A Highly Crystalline Anthracene-Based MOF-74 Series Featuring Electrical Conductivity and Luminescence. ChemRxiv. Preprint.

Recently, a small group of metal-organic frameworks (MOFs) has been discovered featuring substantial charge transport properties and electrical conductivity, hence promising to broaden the scope of potential MOF applications in fields such as batteries, fuel cells and supercapacitors. In combination with light emission, electroactive MOFs are intriguing candidates for chemical sensing and optoelectronic applications. Here, we incorporated anthracene-based building blocks into the MOF-74 topology with five different divalent metal ions, that is, $\mathrm{Zn2}+, \mathrm{Mg} 2+, \mathrm{Ni2}+, \mathrm{Co} 2+$ and $\mathrm{Mn} 2+$, resulting in a series of highly crystalline MOFs, coined ANMOF-74(M). This series of MOFs features substantial photoluminescence, with ANMOF-74(Zn) emitting across the whole visible spectrum. The materials moreover combine this photoluminescence with high surface areas and electrical conductivity. Compared to the original MOF-74 materials constructed from 2,5-dihydroxy terephthalic acid and the same metal ions $\mathrm{Zn} 2+, \mathrm{Mg} 2+, \mathrm{Ni} 2+, \mathrm{Co} 2+$ and $\mathrm{Mn} 2+$, we observed a conductivity enhancement of up to six orders of magnitude. Our results point towards the importance of building block design and the careful choice of the embedded MOF topology for obtaining materials with desired properties such as photoluminescence and electrical conductivity.

File list (2)

ANMOF_MAIN.pdf (1.34 MiB) view on ChemRxiv - download file ANMOF_SI.pdf (2.57 MiB) view on ChemRxiv • download file 


\section{A highly Crystalline Anthracene-based MOF-74 Series featuring Elec- trical Conductivity and Luminescence}

Patricia I. Scheurle ${ }^{\dagger \nabla}$, Andre Mähringer ${ }^{\dagger \dagger}$, Andreas C. Jakowetz ${ }^{\dagger \nabla}$, Pouya Hosseini ${ }^{\infty}$, Alexander F. Rich$\operatorname{ter}^{\nabla \perp}$, Gunther Wittstock ${ }^{\infty}$, Dana D. Medina ${ }^{\dagger *}$ and Thomas Bein ${ }^{\dagger *}$

\section{AUTHOR ADDRESSES}

†Department of Chemistry, Ludwig-Maximilians-Universität (LMU), Butenandtstr. 5-13 (E), 81377 Munich, Germany.

${ }^{\perp}$ Chair for Photonics and Optoelectronics, Nano-Institute Munich, Department of Physics, Ludwig-Maximilians-Universität (LMU), Königinstr. 10, 80539 Munich, Germany.

${ }^{\nabla}$ Nanosystems Initiative Munich (NIM) and Center for NanoScience (CeNS), Schellingstr. 4, $80799 \mathrm{Mu}-$ nich, Germany.

${ }^{\infty}$ Institute of Chemistry, Carl von Ossietzky Universität Oldenburg, 26111 Oldenburg, Germany.

ABSTRACT: Recently, a small group of metal-organic frameworks (MOFs) has been discovered featuring substantial charge transport properties and electrical conductivity, hence promising to broaden the scope of potential MOF applications in fields such as batteries, fuel cells and supercapacitors. In combination with light emission, electroactive MOFs are intriguing candidates for chemical sensing and optoelectronic applications. Here, we incorporated anthracene-based building blocks into the MOF-74 topology with five different divalent metal ions, that is, $\mathrm{Zn}^{2+}$, $\mathrm{Mg}^{2+}, \mathrm{Ni}^{2+}, \mathrm{Co}^{2+}$ and $\mathrm{Mn}^{2+}$, resulting in a series of highly crystalline MOFs, coined ANMOF-74(M). This series of MOFs features substantial photoluminescence, with ANMOF-74(Zn) emitting across the whole visible spectrum. The materials moreover combine this photoluminescence with high surface areas and electrical conductivity. Compared to the original MOF-74 materials constructed from 2,5-dihydroxy terephthalic acid and the same metal ions $\mathrm{Zn}^{2+}, \mathrm{Mg}^{2+}, \mathrm{Ni}^{2+}, \mathrm{Co}^{2+}$ and $\mathrm{Mn}^{2+}$, we observed a conductivity enhancement of up to six orders of magnitude. Our results point towards the importance of building block design and the careful choice of the embedded MOF topology for obtaining materials with desired properties such as photoluminescence and electrical conductivity.

KEYWORDS: Metal-organic frameworks, MOF-74 topology, electrical conductivity, luminescence, anthracene 
Metal-organic frameworks (MOFs) are composed of metal ions or clusters that are interconnected by rigid organic building blocks forming crystalline porous structures. ${ }^{1}$ Based on their well-defined and tunable pore spaces and high surface areas, many MOFs are attractive candidates for gas storage separation and storage applications. ${ }^{2,3}$ Depending on the organic building blocks and the respective metal species, MOFs can be synthesized with diverse chemical composition, connectivity and functionality. Therefore, a large variety of different chemical and physical properties can be attained. ${ }^{4,5,6}$ Recently, the intriguing property of electrical conductivity was added to this portfolio. ${ }^{2,7-12}$ Hereby, an increasing number of MOFs were investigated with a view on applications in the fields of charge storage, semiconductors, chemical sensing, energy conversion or electrocatalysis. ${ }^{12-20}$

To date, only few MOFs have been reported exhibiting the desired property of electrical conductivity in combination with ultrahigh surface area, which is viewed to be essential for sensing or charge storage applications. ${ }^{21}$ Recently, structures based on the MOF-74 topology constructed with 2,5-dihydroxy terephthalic acid were introduced as electrically conductive platforms. ${ }^{2,5,22,23}$ MOF-74 isostructures connected through phenoxide-metal nodes showed low conductivity values, whereas structures containing sulfur ligating functionalities in the linking motif or mixed valance of the metal ions showed an increased electrical conductivity of up to $10^{-6} \mathrm{~S} \mathrm{~cm}^{-1} \cdot 2,24$

Here, we present a series of MOF-74 analogs comprising an anthracene core in the linear organic linker. Anthracene and its derivatives are known for their high charge carrier mobility and electroluminescent properties. ${ }^{11,21}$ We demonstrate that the incorporation of anthracene-containing building blocks can strongly enhance electrical conductivity in phenoxide-connected MOF-74 topologies. Furthermore, we show that the anthracene core endows the MOFs with photoluminescent properties allowing for the realization of light emitting and electrically conducting MOFs. For constructing the framework, we first established the synthesis of an anthracene-based building block modified with ortho-hydroxy para-carboxylic functionalities enabling the required bonding to metal ions, namely 4,4'-(anthracene-9,10-diyl)bis(2hydroxybenzoic acid), ABHB. 
Using the anthracene building blocks and divalent metal ions, that is, $\mathrm{Zn}^{2+}, \mathrm{Mg}^{2+}, \mathrm{Ni}^{2+}, \mathrm{Co}^{2+}$ and $\mathrm{Mn}^{2+}$, five novel, highly crystalline and porous anthracene-based MOF-74-type (ANMOF-74) materials were synthesis under solvothermal conditions (Figure 1). The ANMOF-74 series features an enhancement of electrical conductivity by several orders of magnitude compared to the respective original MOF-74 isostructures. Furthermore, the photoluminescence life time and quantum yield were determined for the ANMOF-74 series.

A

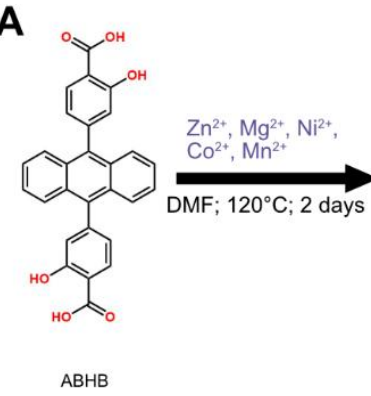

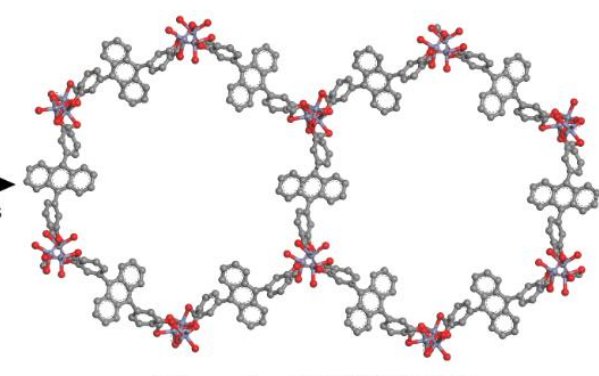

Anthracene based MOF-74 (ANMOF-74)

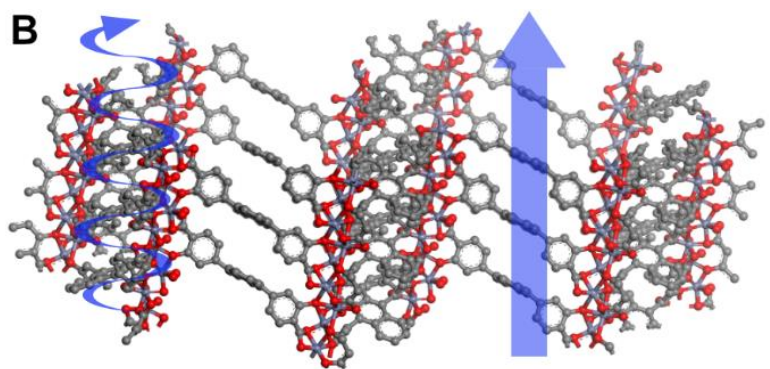

Anthracene stacks along c-axis

Figure 1. A) Synthesis scheme of the anthracene-based MOF-74 (ANMOF-74). The organic building block ABHB forms porous hexagonal frameworks with the respective metal ions. B) View on the helical metal-oxo chains of the ANMOF-74 structure (left) and the anthracene moieties (right) stacked along the crystallographic $c$-direction.

\section{RESULTS AND DISCUSSION}

The ABHB linker used here was synthesized via a three-step reaction procedure (see Schemes S1S3). ${ }^{25,26}$ In short, the reaction of methyl 4-iodosalicylate and bis(pinacolato)diboron afforded a boronated intermediate product, which was subsequently coupled in a Suzuki reaction with the brominated anthracene core to the methyl protected ABHB linking motif. After deprotection and purification, the final organic building block ABHB was obtained in sufficient yields (ca. 70\%) as a beige powder. For the MOF synthesis, the stoichiometric reaction of $\mathrm{ABHB}$ with the respective metal precursors $\left(\mathrm{Zn}^{2+}, \mathrm{Mg}^{2+}, \mathrm{Ni}^{2+}\right.$, $\mathrm{Co}^{2+}$ and $\mathrm{Mn}^{2+}$ ) in different $\mathrm{DMF} / \mathrm{MeOH} / \mathrm{EtOH} / \mathrm{BnOH} / \mathrm{H}_{2} \mathrm{O}$ mixtures at $120{ }^{\circ} \mathrm{C}$ for 2 days led to the precipitation of colored powders. Here, the color of the obtained powders was strongly dependent on the 
utilized metal ion precursor (for further information see SI). The obtained powders were washed with DMF and methanol and subsequent dried under dynamic vacuum.
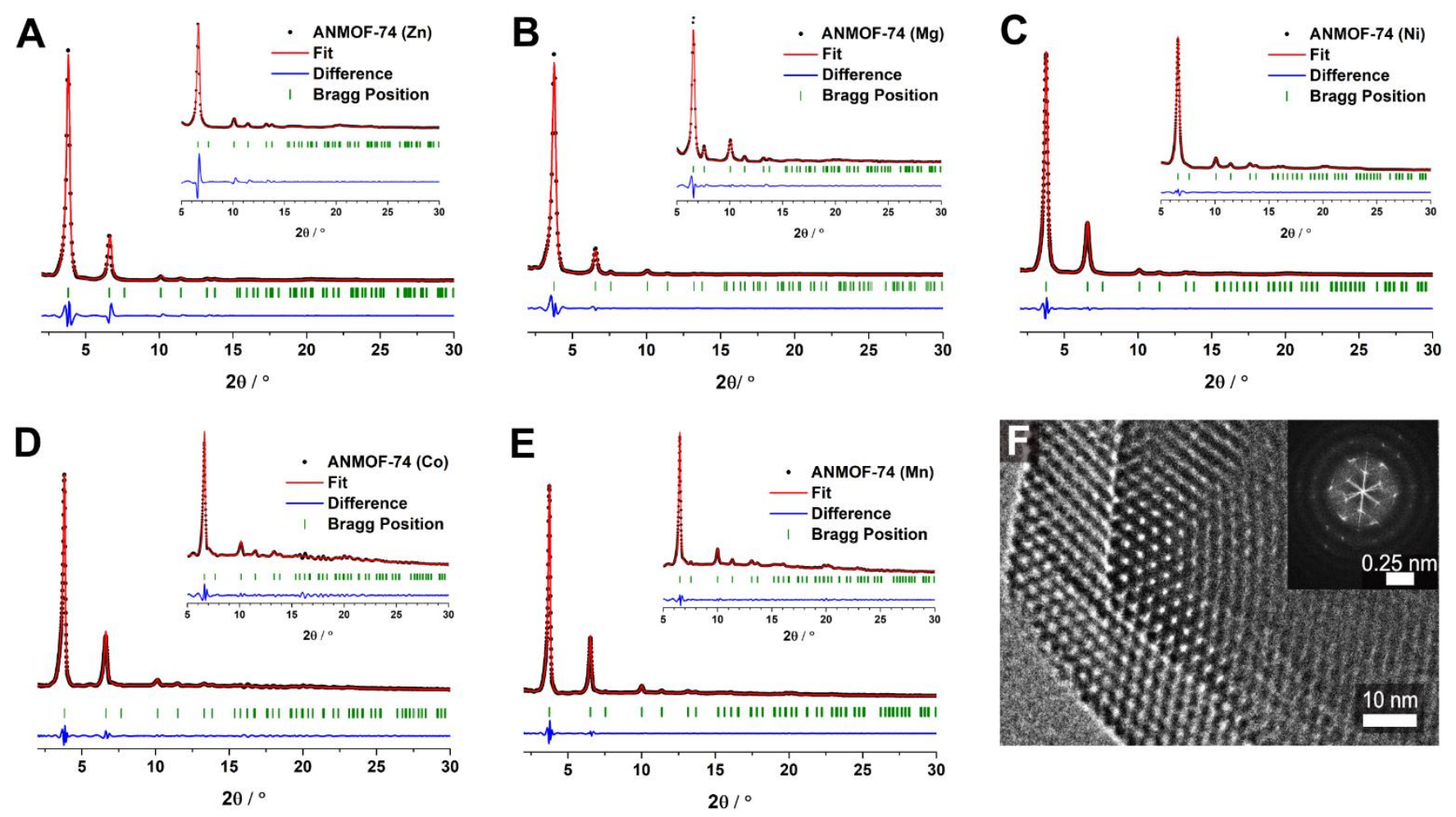

Figure 2. Experimental powder X-ray diffraction (PXRD) patterns of the ANMOF-74(M) series showing (A) the Zn-, (B) Mg-, (C) Ni-, (D) Co- and (E) Mn-MOF derivatives, respectively (black). The simulated PXRD pattern was Pawley-refined (red) according to the experimental data, whereby the difference plot is depicted in blue. The respective Bragg positions of the predicted hexagonal unit cell are shown as green bars. (F) A high resolution TEM image of an ANMOF-74 (Co) crystal oriented along the [001] axis with FFT of the entire image as inset.

Powder X-ray diffraction (PXRD) data of the dried powders are shown in Figure 2. All materials exhibit a high degree of crystallinity with pronounced reflections at $3.75^{\circ}, 6.51^{\circ}, 10.02^{\circ}, 11.34^{\circ}, 13.14^{\circ}$ and $13.66^{\circ} 2 \theta$. Bragg reflections at up to $25^{\circ} 2 \theta$ could be detected, underlining the high crystallinity of 
the obtained ANMOF-74 series. The collected diffractograms matched with the ones predicted from simulated model structures. The predicted model structures were obtained with Accelrys Materials Studio 7.0 using the calculated MOF-74 structure as a model. ${ }^{2}$ After establishing the simulated unit cell, a PXRD pattern was calculated, which was Pawley-refined according to the respective experimental PXRD patterns $\left(\mathrm{R}_{\mathrm{wp}}\right.$ ranging from 2.09-4.09\%). From the refinement, we conclude that the ANMOF-74 system crystallizes in the trigonal space group $R 3$ with $a=b=46.3 \AA$, c $=5.7 \AA, \alpha=\beta=90^{\circ}$ and $\gamma=120^{\circ}$. These findings are in good agreement with simulations performed for related MOF-74 structures. ${ }^{11,27}$ All ANMOF-74 materials exhibit one-dimensional hexagonal channels with pore apertures of $2 \mathrm{~nm}$. These channels are formed by the ABHB units that are interconnected in the $c$-direction by infinite helical metaloxo chains. ANMOF-74 further includes infinite stacks of the anthracene core arranged along the $c$-direction, with the distance between adjacent anthracene cores estimated to be $5.7 \AA$ (Figure 1B). For the ABHB unit, we determined a torsion angle of $60.7^{\circ}$ between the benzene rings and the anthracene core. Transmission electron microscopy (TEM) analysis of the ANMOF-74 series revealed highly crystalline materials (Figure S2). For ANMOF-74(Co), hexagonally shaped crystal facets and large cross-section domain sizes of about $120 \mathrm{~nm}$ illustrate the high degree of crystallinity (Figure 2F). The calculated diffraction pattern in the fast Fourier transform (FFT) of the TEM image shows angles of $120^{\circ}$ in accordance with the proposed trigonal crystal system.

The synthesized powders were further investigated using X-ray photoelectron spectroscopy (XPS). As summarized in Figures S12-S16, XPS spectra of ANMOF-74(M) show the presence of the respective metal, $\mathrm{C}$ and $\mathrm{O}$ peaks attributed to the MOFs, as well as nitrogen, tentatively attributed to DMF residues coordinating to the metal ions in the structure. These findings are in accordance with the highresolution analysis of the $\mathrm{O} 1 \mathrm{~s}$ regions in the XPS spectra of all ANMOFs, indicating three types of oxygen atoms. The signals can be assigned to the functional groups (carboxy and hydroxy) of the ligating building block and to the oxygen related to DMF. Only ANMOF-74(Zn) has a second $\mathrm{N}$ 1s peak arising from residual $\mathrm{NO}_{3}{ }^{-}$, which apparently originates from the $\mathrm{Zn}\left(\mathrm{NO}_{3}\right)_{2}$ synthesis precursor. Importantly, $\mathrm{Cl}^{-}$or 
additional $\mathrm{N}$ peaks related to impurities originating from the metal precursors were not observed for all other samples of the ANMOF-74 series. The purity of the obtained materials was further studied by energy dispersive X-ray spectroscopy (EDX) which showed no impurities arising from precursor anions (see Figure S11). Thermogravimetric analysis (TGA) revealed that the ANMOF-74(M) materials were not decomposed below $300{ }^{\circ} \mathrm{C}$ under dynamic conditions (Figure S10).
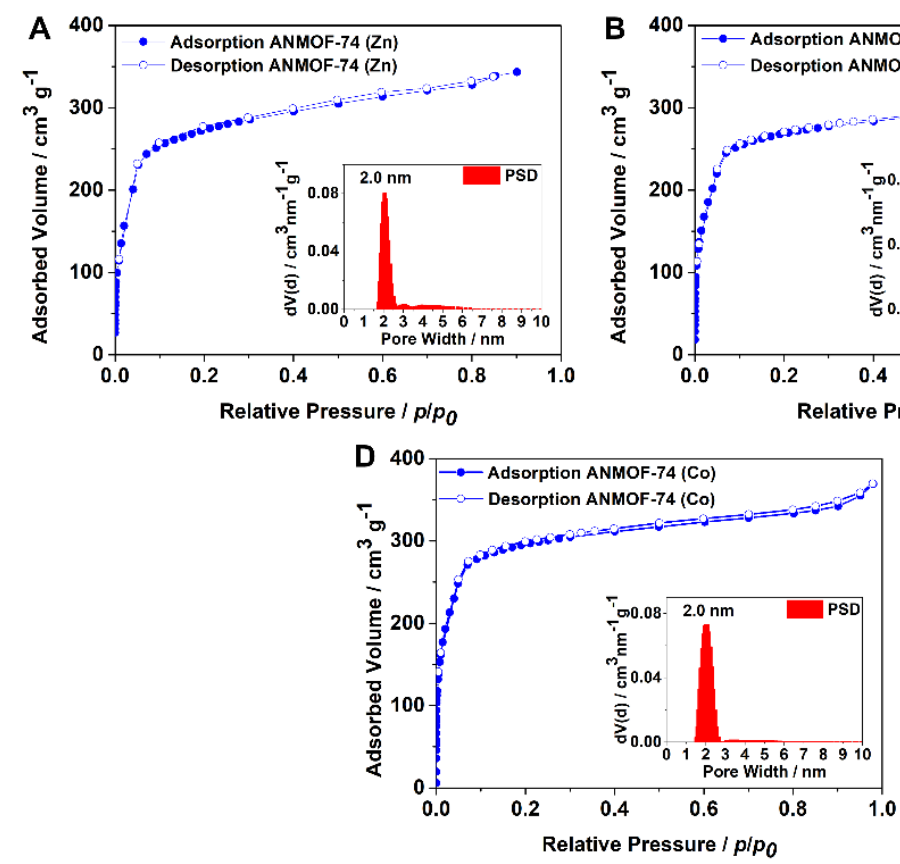
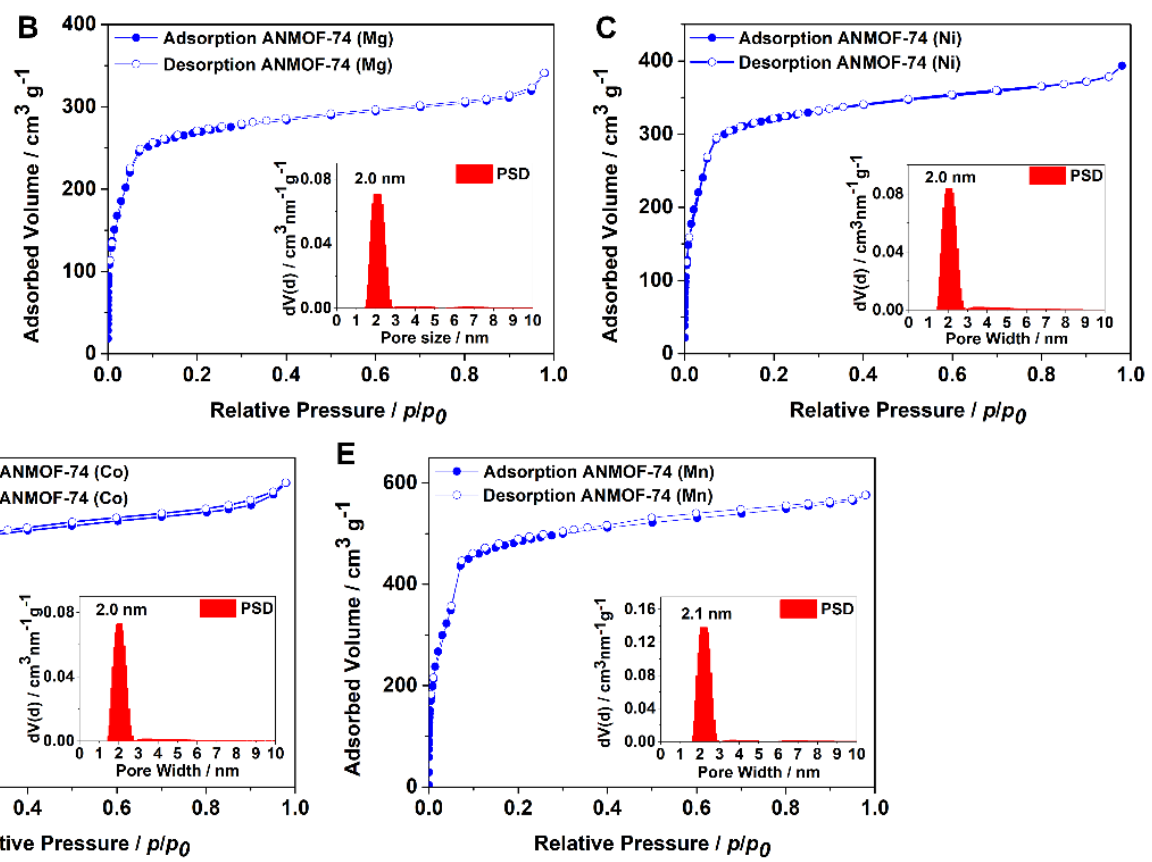

Figure 3. Nitrogen physisorption isotherms of (A) ANMOF-74(Zn), (B) ANMOF-74(Mg), (C) ANMOF74(Ni), (D) ANMOF-74(Co) and (E) ANMOF-74(Mn) showing Type I(b) shape. The ad- and desorption branches are depicted as blue and white colored circles, respectively. Pore size distributions (PSD) are included as insets.

The pore accessibility of the activated samples was investigated by nitrogen physisorption experiments. The nitrogen isotherms $(77 \mathrm{~K})$ of all ANMOF-74(M) powders (Figure 3) show IUPAC Type I(b) ${ }^{28}$ sorption behavior, indicating that the ANMOF-74(M) form large micropores $(<2.5 \mathrm{~nm})$. All examined MOFs show an initial uptake of about $200 \mathrm{~cm}^{3} \mathrm{~g}^{-1}$ at low pressures and a second uptake at $0.02-0.05 \mathrm{p} / \mathrm{p}_{0}$ of about $100 \mathrm{~cm}^{3} \mathrm{~g}^{-1}$. The adsorption branch and the desorption branch merge at low relative pressures 
illustrating a reversible process. The Brunauer-Emmett-Teller (BET) surface areas and total pore volumes for the ANMOF-74(M) bulk materials series were calculated to be $1124 \mathrm{~m}^{2} \mathrm{~g}^{-1}$ and $0.49 \mathrm{~cm}^{3} \mathrm{~g}^{-1}$ for $\mathrm{Zn}$, $1137 \mathrm{~m}^{2} \mathrm{~g}^{-1}$ and $0.48 \mathrm{~cm}^{3} \mathrm{~g}^{-1}$ for $\mathrm{Mg}, 1352 \mathrm{~m}^{2} \mathrm{~g}^{-1}$ and $0.56 \mathrm{~cm}^{3} \mathrm{~g}^{-1}$ for $\mathrm{Ni}, 1213 \mathrm{~m}^{2} \mathrm{~g}^{-1}$ and $0.53 \mathrm{~cm}^{3} \mathrm{~g}^{-1}$ for Co, $1748 \mathrm{~m}^{2} \mathrm{~g}^{-1}$ and $0.83 \mathrm{~cm}^{3} \mathrm{~g}^{-1}$ for $\mathrm{Mn}$, respectively. The pore size distributions were calculated using quenched solid density functional theory (QSDFT), giving pore sizes of $2.1 \mathrm{~nm}$ in ANMOF-74(Mn) and $2.0 \mathrm{~nm}$ in all other ANMOF-74(M) structures, which match the predicted value of $2.0 \mathrm{~nm}$ obtained by the simulated structure. Connolly surface area calculations with nitrogen as a probe molecule $(2.6 \AA)$ predict a theoretical surface area of $1757 \mathrm{~m}^{2} \mathrm{~g}^{-1}$, which is matched best by the BET results for the ANMOF74(Mn) sample.

Scanning electron microscopy (SEM) images of the ANMOF-74(M) powders reveal needleshaped crystallites forming spherical agglomerates (Figures 4 and S2). In addition, high magnification SEM analysis reveals crystallites with a well-faceted hexagonal cross-section, further confirming the proposed trigonal crystal structures. 


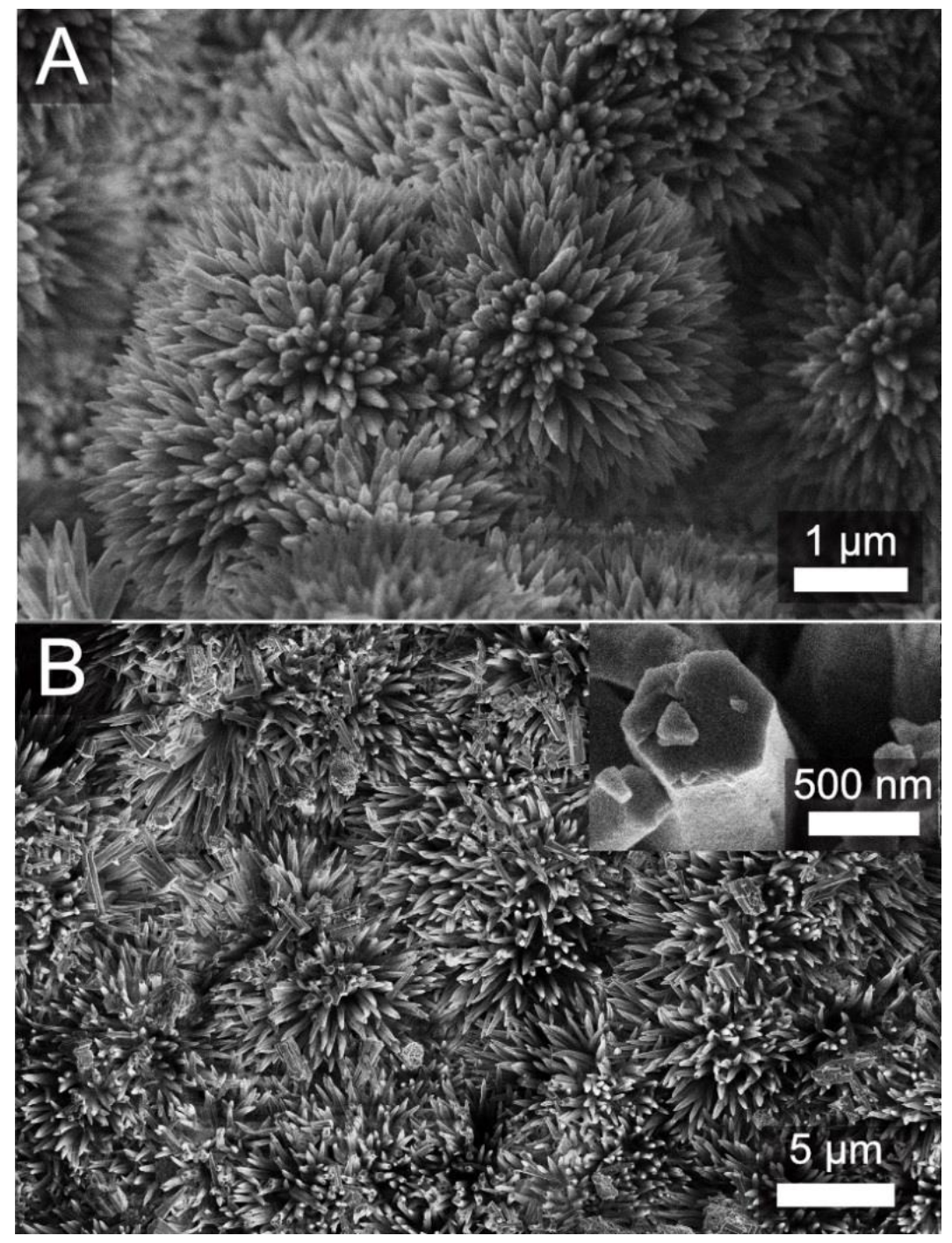

Figure 4. (A) and (B) SEM images of the ANMOF-74 bulk materials (A) ANMOF-74(Ni) (B) ANMOF74(Mn) with a high magnification of a hexagonally shaped crystal depicted as inset.

To elucidate the electrical properties of the ANMOF-74 series, we performed four-point probe van der Pauw measurements of crystalline and pelleted samples (for further details see SI). The obtained results are summarized in Table 1. 
Table 1. Electrical conductivity values of ANMOF-74(M) and MOF-74(M) samples obtained with pellets by using the four-probe van der Pauw technique.

\begin{tabular}{|c|c|c|c|c|c|}
\hline ANMOF-74 (Zn) & MOF-74 (Zn) & ANMOF-74 (Mg) & MOF-74 (Mg) & ANMOF-74 (Ni) & MOF-74 (Ni) \\
\hline $6 \times 10^{-8} \mathrm{~S} \mathrm{~cm}^{-1}$ & $6 \times 10^{-12} \mathrm{~S} \mathrm{~cm}^{-1}$ & $5 \times 10^{-9} \mathrm{~S} \mathrm{~cm}^{-1}$ & $6 \times 10^{-13} \mathrm{~S} \mathrm{~cm}^{-1}$ & $4 \times 10^{-7} \mathrm{~S} \mathrm{~cm}^{-1}$ & $6 \times 10^{-13} \mathrm{~S} \mathrm{~cm}^{-1}$ \\
\hline ANMOF-74 (Co) & MOF-74 (Co) & ANMOF-74 (Mn) & MOF-74 (Mn) & & \\
\hline $4 \times 10^{-8} \mathrm{~S} \mathrm{~cm}^{-1}$ & $6 \times 10^{-13} \mathrm{~S} \mathrm{~cm}^{-1}$ & $3 \times 10^{-8} \mathrm{~S} \mathrm{~cm}^{-1}$ & $4 \times 10^{-13} \mathrm{~S} \mathrm{~cm}^{-1}$ & & \\
\hline
\end{tabular}

The values obtained for ANMOF-74(M) range from $10^{-7}$ to $10^{-9} \mathrm{~S} \mathrm{~cm}^{-1}$, corresponding to a striking increase of up to six orders of magnitude with regard to the values obtained (and reported) for the respective MOF-74. To validate the impact of the anthracene core on the electrical conductivity, crystalline MOF-74 powders with the same set of metal ions were synthesized, activated (vacuum dried), compressed into pellets and their electrical conductivity was evaluated. The van der Pauw measurements of the MOF74 pellets confirmed the ANMOF-74 series to be substantially more conductive than the MOF-74 isostructures. We attribute the enhanced electrical conductivity to the presence of the electron-rich anthracene core incorporated in the ANMOF-74, in contrast to the rather electron-poor 2,5-dihydroxy terephthalic acid building block embedded in the MOF-74 series. Here, our strategy of embedding the electronrich anthracene-based linker showed a remarkable increase in conductivity, however, the measured values are still modest compared to other conducting MOF platforms..$^{29,30}$ Due to the fairly large stacking distance between adjacent anthracene units we postulate that charge transport still occurs mostly through the metaloxo backbone by charge carrier hopping between metal nodes.

To further elucidate the impact of the MOF-embedded anthracene moiety, we examined the optical properties of the ANMOF-74(M) bulk materials by using UV-vis absorption and photoluminescence (PL) spectroscopy, summarized in Figure 5. The UV-vis spectra were measured in diffusive reflectance geom- 
etry and transformed using the Kubelka-Munk equation. For all examined ANMOF-74 samples the absorption bands are located in the UV and blue spectral regions. ANMOF-74(Zn) has an absorption onset of around $528 \mathrm{~nm}$ and a second one at around $450 \mathrm{~nm}$, and ANMOF-74(Mg; Ni; Co; Mn) at around $440 \mathrm{~nm}$. Tauc plots were analyzed, assuming direct band gaps, yielding an optical band gap of $2.86 \mathrm{eV}$ for ANMOF-74( $\mathrm{Zn})$ and in case of ANMOF-74(Mg; Ni; Co; Mn) band gaps between $2.91 \mathrm{eV}$ and $2.95 \mathrm{eV}$ (Figure S5). These five MOFs have two sharp absorption maxima at $400 \mathrm{~nm}$ and $380 \mathrm{~nm}$, which originate from the integrated ABHB building blocks (see Figure S4 (A)).

Photoluminescence (PL) of the ANMOF-74(M) bulk materials was measured with a pulsed 378 $\mathrm{nm}$ excitation under argon atmosphere to protect the anthracene from degradation. The PL spectrum of ANMOF-74(Zn) shows a very broad emission band over the whole visible spectrum with a maximum at $570 \mathrm{~nm}$ and a large Stokes shift of $170 \mathrm{~nm}$. ANMOF-74(Mg) and ANMOF-74(Co), as well as ANMOF74(Ni) and ANMOF-74(Mn) have a narrow emission band with a small shoulder and an emission maximum between $438 \mathrm{~nm}$ and $444 \mathrm{~nm}$, respectively. It is similar to the emission band at $441 \mathrm{~nm}$ of the ABHB linker monomer measured in solution (see Figure S4 (B)). These MOFs exhibit smaller Stokes shifts of 38 - $44 \mathrm{~nm}$. ANMOF-74(Co) and ANMOF-74(Ni) also have a weaker emission band at $692 \mathrm{~nm}$. ANMOF-74(Mn) has a weak emission band at $701 \mathrm{~nm}$. 

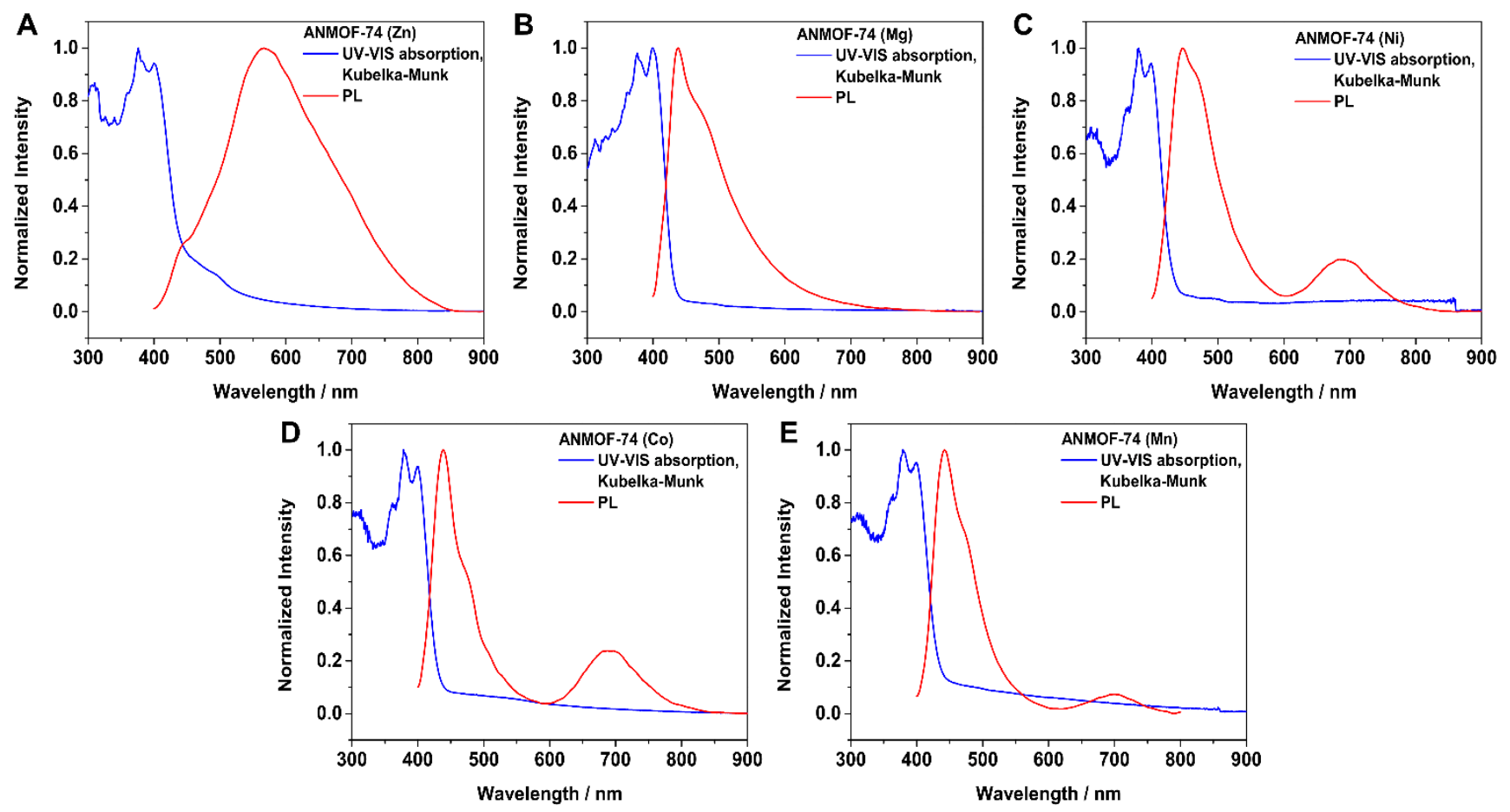

Figure 5. UV-VIS absorption measured in diffusive reflectance of the MOF powders dispersed with $\mathrm{BaSO}_{4}$ (blue line) and the photoluminescence (PL) spectra of the neat MOF bulk materials measured with pulsed $378 \mathrm{~nm}$ excitation (red line): A) ANMOF-74(Zn), B) ANMOF-74(Mg), C) ANMOF-7(Ni), D) ANMOF-74(Co) and E) ANMOF-74(Mn).

Time-correlated single photon counting (TCSPC) traces of ANMOF-7(Zn) and ANMOF-74(Mg) were recorded at the respective emission maxima of $570 \mathrm{~nm}$ and $438 \mathrm{~nm}$, shown in Figure 6. Both MOFs show very similar relaxation behavior, analyzed with a triexponential fit. Both exhibit fast decays of around $\tau_{1}=0.16 \mathrm{~ns}$, which is near the instrumental response limit. The medium component has a lifetime $\tau_{2}$ of $0.79 \mathrm{~ns}$ and $1.03 \mathrm{~ns}$ while the third component has a lifetime $\tau_{3}$ of $3.44 \mathrm{~ns}$ and $4.90 \mathrm{~ns}$ for $\mathrm{Zn}$ and $\mathrm{Mg}$, respectively. The main difference between the two materials is the fractional distribution of lifetimes. While the medium-lifetime decay channel is contributing almost $60 \%$ to the overall emission in the ANMOF-74(Zn) and only 50\% in the ANMOF-74(Mg), this difference is balanced by the longer lifetime components, i.e. $18 \%$ versus $29 \%$ in the $\mathrm{Zn}$ and $\mathrm{Mg}$ ANMOFs, respectively. This results in the faster 
overall decay of the ANMOF-74(Zn) as compared to the longer-lived ANMOF-7(Mg). Due to the bright emission and long lifetime of ANMOF-74(Mg), we also measured the photoluminescence quantum yield (PLQY), which reaches 2.5\%. Quantum efficiency of the ABHB linker molecules as solid powder show PLQY values of up to $13 \%$, which is reduced by one order of magnitude upon incorporation into the framework. However, the luminescence is still detectable with the naked eye by irradiating the MgANMOF sample with a UV lamp.

TCSPC traces of ANMOF-74(Ni), ANMOF-74(Co) and ANMOG-74(Mn) show similar lifetime components with $\tau_{1}$ around $0.06 \mathrm{~ns}, \tau_{2}$ between $0.79 \mathrm{~ns}$ and $0.89 \mathrm{~ns}$ and $\tau_{3}$ in the range of $5-7 \mathrm{~ns}$ (see Figure S6). The ABHB linker monomer has a monoexponential decay with a lifetime of 4.3 ns (see S6). The long PL lifetime of ANMOF-74(Mg) in comparison to the other ANMOFs might be related to the different emission spectrum. ANMOF-74(Mg), similar to the ABHB linker, does not have a second emission band at around $700 \mathrm{~nm}$. But the PL lifetime of ANMOF-74(Mg) and of the ABHB linker are much longer compared to the other ANMOFs. Thus, in the ANMOF-74(Mg), the linkers might be similarly electronically 'isolated as the monomer in solution while the emission band at around $700 \mathrm{~nm}$ might be related to electronic interactions between linkers (and transition metal ions), effectively decreasing the PL lifetime. 


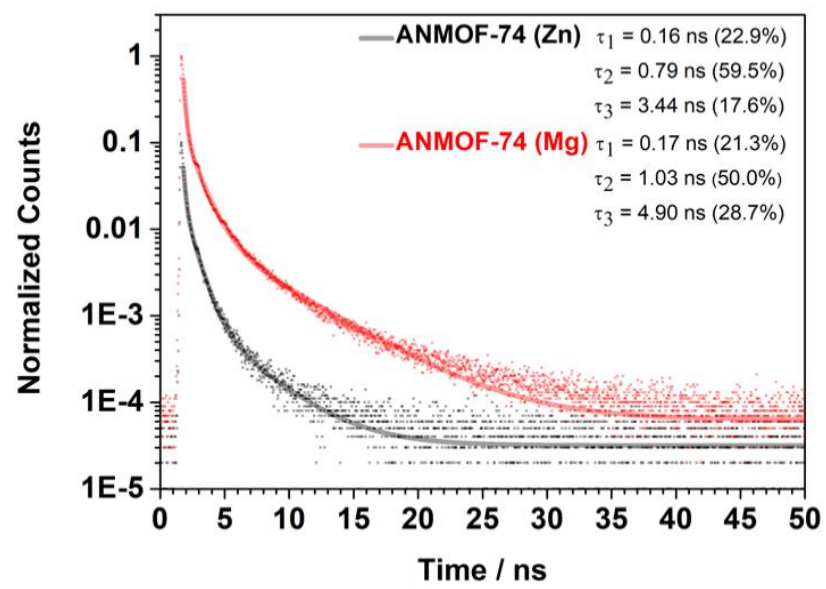

Figure 6. The time-correlated single photon counting (TCSPC) traces corresponding to the PL spectra of ANMOF-74(Zn) (black) and ANMOF-74(Mg) (red), which were recorded at the respective emission maximum of each MOF. The lifetimes were obtained from triexponential fits (solid lines) to the data (scatter). The fractions of emitted photons corresponding to the respective lifetimes are stated in brackets.

\section{CONCLUSIONS}

In our study, we present the successful synthesis of a novel series of metalorganic frameworks showing MOF-74 topology, coined ANMOF-74. The frameworks are built from anthracene-based linking motifs (ABHB) connected via helical metal-oxo chains, whereby a range of different metal ions could be incorporated ( $\mathrm{Zn}, \mathrm{Mg}, \mathrm{Co}, \mathrm{Ni}$ and $\mathrm{Mn}$ ) into the framework. All ANMOF-74 materials feature a high degree of crystallinity and show BET surface areas exceeding $1000 \mathrm{~m}^{2} \mathrm{~g}^{-1}$. Furthermore, all ANMOF-74 materials exhibit an enhancement of electrical conductivity of up to one-million fold compared to regular MOF-74. The ANMOF-74 materials feature direct optical band gaps of around $2.9 \mathrm{eV}$ and most show photoluminescence in the blue spectral region. In contrast to the other derivatives, the ANMOF-74(Mg) shows a rather high quantum efficiency of 2.5\%. Remarkably, ANMOF-74(Zn) emits photoluminescence 
across the entire visible spectrum. We believe that this promising combination of high crystallinity, defined porosity, luminescence as well as electrical conductivity embedded in a fixed MOF matrix will enable a range of possible applications such as sensing and charge storage. Our study highlights the great versatility of the MOF-74 topology, allowing for the integration of sterically demanding organic linker structures with interesting electrical and optical properties. In the future, we envision the integration of additional chromophores with interesting optoelectronic properties and a tunable degree of electronic coupling into this highly attractive MOF topology, to further broaden the field of MOF-74 based structures for optoelectronic applications.

\section{ASSOCIATED CONTENT}

\section{Supporting Information}

Supporting Information is provided for the experimental details of the characterization techniques, the synthesis of all compounds and preparation of the samples and characterization using SEM, TEM, UVvis absorption, PL, TGA, EDX and XPS.

\section{AUTHOR INFORMATION}

\section{Corresponding Author}

*Email: bein@1mu.de

*Email: dana.medina@cup.uni-muenchen.de

\section{Present Addresses}

$\dagger$ If an author's address is different than the one given in the affiliation line, this information may be included here.

\section{Author Contributions}

$\ddagger$ P. S. and A. M. contributed equally. The manuscript was written through contributions of all authors. All authors commented on the manuscript.

\section{Funding Sources}

German Science Foundation (SPP 1928, COORNETs, Excellence Clusters NIM and e-conversion), Free State of Bavaria (Solar Technologies Go Hybrid).

\section{ACKNOWLEDGMENT}

The authors are grateful for financial support from the Deutsche Forschungsgemeinschaft (DFG) in the context of the National Research Network COORNETs (SPP 1928), the Excellence Clusters "Nanosystems Initiative Munich 
(NIM)" and "e-conversion", and from the Free State of Bavaria through the Research Network "Solar Technologies go Hybrid”. We also thank Dr. Markus Döblinger for the TEM images and Julian Rotter for the SEM images. Furthermore, we thank Tina Reuther for TGA and sorption measurements.

\section{ABBREVIATIONS}

ANMOF-74, anthracene-based metal-organic framework-74; BET, Brunauer-Emmett-Teller; BnOH, benzyl alcohol; DMF, diemthylformamide; EtOH, ethanol; FFT, fast Fourier transform; PXRD, powder X-ray diffraction; SEM, scanning electron microscopy; $\mathrm{MeOH}$, methanol; TEM, transmission electron microscopy; SBU, secondary building unit, PL, photoluminescence, PLQY, photoluminescence quantum yield; TCSPC, time-correlated single photon counting; TGA, thermogravimetric analysis; UV-VIS spectroscopy, ultraviolet-visible spectroscopy; PSD, pore size distribution; XPS, X-ray photoelectron spectroscopy.

\section{References}

(1) Yaghi, O. M.; Li, H. Hydrothermal Synthesis of a Metal-Organic Framework Containing Large Rectangular Channels. J. Am. Chem. Soc. 1995, 10401-10402.

(2) Sun, L.; Hendon, C. H.; Minier, M. A.; Walsh, A.; Dincă, M. Million-Fold Electrical Conductivity Enhancement in Fe2(DEBDC) versus Mn2(DEBDC) (E = S, O). J. Am. Chem. Soc. 2015, 137, 6164-6167.

(3) Meek, S. T.; Greathouse, J. A.; Allendorf, M. D. Metal-Organic Frameworks: A Rapidly Growing Class of Versatile Nanoporous Materials. Adv. Mater. 2011, 23, 249-267.

(4) Wang, L. J.; Deng, H.; Furukawa, H.; Gándara, F.; Cordova, K. E.; Peri, D.; Yaghi, O. M. Synthesis and Characterization of Metal-Organic Framework-74 Containing 2, 4, 6, 8, and 10 Different Metals. Inorg. Chem. 2014, 53, 5881-5883.

(5) Kapelewski, M. T.; Geier, S. J.; Hudson, M. R.; Stück, D.; Mason, J. A.; Nelson, J. N.; Xiao, D. J.; Hulvey, Z.; Gilmour, E.; FitzGerald, S. A. et al. M2(m-dobdc) (M = Mg, Mn, Fe, Co, Ni) metal-organic frameworks exhibiting increased charge density and enhanced $\mathrm{H} 2$ binding at the open metal sites. J. Am. Chem. Soc. 2014, 136, 12119-12129.

(6) Rosi, N. L.; Kim, J.; Eddaoudi, M.; Chen, B.; O'Keeffe, M.; Yaghi, O. M. Rod packings and metal-organic frameworks constructed from rod-shaped secondary building units. J. Am. Chem. Soc. 2005, 127, 1504-1518.

(7) Usman, M.; Mendiratta, S.; Batjargal, S.; H., G.; Haider, M.; Rao Gade, N.; Chen, J.-W.; Chen, Y.-F.; Lu, K.-L. Semiconductor Behavior of a Three-Dimensional Strontium-Based Metal-Organic Framework. ACS Appl. Mater. Interfaces 2015, 7, 22767-22774.

(8) Sun, L.; Campbell, M. G.; Dincă, M. Electrically Conductive Porous Metal-Organic Frameworks. Angew. Chem. 2016, 55, 3566-3579.

(9) Usman, M.; Mendiratta, S.; Lu, K.-L. Semiconductor Metal-Organic Frameworks: Future Low-Bandgap Materials. Adv. Mater. 2017, 29, 1605071.

(10) Kobayashi, Y.; Jacobs, B.; Allendorf, M. D.; Long, J. R. Conductivity, Doping, and Redox Chemistry of a Microporous Dithiolene-Based Metal-Organic Framework. Chem. Mater. 2010, 22, 4120-4122.

(11) Guo, Z.; Panda, D. K.; Gordillo, M. A.; Khatun, A.; Wu, H.; Zhou, W.; Saha, S. Lowering Band Gap of an Electroactive Metal-Organic Framework via Complementary Guest Intercalation. ACS Appl. Mater. Interfaces 2017, 9, 32413-32417. 
(12) Stavila, V.; Talin, A. A.; Allendorf, M. D. MOF-based electronic and opto-electronic devices. Chem. Soc. Rev. 2014, 43, 5994-6010.

(13) Campbell, M. G.; Sheberla, D.; Liu, S. F.; Swager, T. M.; Dincă, M. Cu(hexaiminotriphenylene)2: an electrically conductive 2D metal-organic framework for chemiresistive sensing. Angew. Chem. 2015, 54, 4349-4352.

(14) Zhang, Z.; Yoshikawa, H.i.; Awaga, K. Monitoring the solid-state electrochemistry of Cu(2,7-AQDC) (AQDC $=$ anthraquinone dicarboxylate) in a lithium battery: coexistence of metal and ligand redox activities in a metal-organic framework. J. Am. Chem. Soc. 2014, 136, 16112-16115.

(15) Campbell, M. G.; Dincă, M. Metal-Organic Frameworks as Active Materials in Electronic Sensor Devices. Sensors 2017, 17, 1106.

(16) Kreno, L. E.; Leong, K.; Farha, O. K.; Allendorf, M.; van Duyne, R. P.; Hupp, J. T. Metal-organic framework materials as chemical sensors. Chem. Rev. 2012, 112, 1105-1125.

(17) Zhu, X.-D.; Zhang, K.; Wang, Y.; Long, W.-W.; Sa, R.-J.; Liu, T.-F.; Lü, J. Fluorescent Metal-Organic Framework (MOF) as a Highly Sensitive and Quickly Responsive Chemical Sensor for the Detection of Antibiotics in Simulated Wastewater. Inorg. Chem. 2018, 57, 1060-1065.

(18) Yi, F.-Y.; Chen, D.; Wu, M.-K.; Han, L.; Jiang, H.-L. Chemical Sensors Based on Metal-Organic Frameworks. ChemPlusChem 2016, 81, 675-690.

(19) Kim, H.; Rao, S. R.; Kapustin, E. A.; Zhao, L.; Yang, S.; Yaghi, O. M.; Wang, E. N. Adsorption-based atmospheric water harvesting device for arid climates. Nat. Commun. 2018, 9, 1191.

(20) Gérard, F.; Millange, F.; Morcrette, M.; Serre, C.; Doublet, M.-L.; Grenèche, J.-M.; Tarascon, J.-M. Mixedvalence li/fe-based metal-organic frameworks with both reversible redox and sorption properties. Angew. Chem.

2007, 46, 3259-3263.

(21) Chen, D.; Xing, H.; Su, Z.; Wang, C. Electrical conductivity and electroluminescence of a new anthracenebased metal-organic framework with $\pi$-conjugated zigzag chains. Chem. Commun. 2016, 52, 2019-2022.

(22) Deng, H.; Grunder, S.; Cordova, K. E.; Valente, C.; Furukawa, H.; Hmadeh, M.; Gándara, F.; Whalley, A. C.; Liu, Z.; Asahina, S. et al. Large-pore apertures in a series of metal-organic frameworks. Science 2012, 336, $1018-1023$.

(23) Cozzolino, A. F.; Brozek, C. K.; Palmer, R. D.; Yano, J.; Li, M.; Dincă, M. Ligand redox non-innocence in the stoichiometric oxidation of Mn2(2,5-dioxidoterephthalate) (Mn-MOF-74). J. Am. Chem. Soc. 2014, 136, $3334-3337$.

(24) Sun, L.; Miyakai, T.; Seki, S.; Dincă, M. Mn2(2,5-disulfhydrylbenzene-1,4-dicarboxylate): a microporous metal-organic framework with infinite (-Mn-S-)o chains and high intrinsic charge mobility. J. Am. Chem. Soc. 2013, 135, 8185-8188.

(25) Fracaroli, A. M.; Furukawa, H.; Suzuki, M.; Dodd, M.; Okajima, S.; Gándara, F.; Reimer, J. A.; Yaghi, O. M. Metal-organic frameworks with precisely designed interior for carbon dioxide capture in the presence of water. J. Am. Chem. Soc. 2014, 136, 8863-8866.

(26) Hirofumi, M.; Akira, S.; Yusuke, M.; Yoshinori, I. Polycarboxylic acid containing condensed aromatic ring, crystalline network complex using same, and gas storage material. WO 2017006638 Al 2017.

(27) Zheng, J.; Vemuri, R. S.; Estevez, L.; Koech, P. K.; Varga, T.; Camaioni, D. M.; Blake, T. A.; McGrail, B. P.; Motkuri, R. K. Pore-Engineered Metal-Organic Frameworks with Excellent Adsorption of Water and Fluorocarbon Refrigerant for Cooling Applications. J. Am. Chem. Soc. 2017, 139, 10601-10604.

(28) Thommes, M.; Kaneko, K.; Neimar K, A. V.; Olivier, J. P.; Rodriguez-Reinoso, F.; Rouquerol, J.; Sing, K. S. W. Physisorption of gases, with special reference to the evaluation of surface area and pore size distribution (IUPAC Technical Report). Pure Appl. Chem. 2015, 87, 1051-1069. 
(29) Ko, M.; Mendecki, L.; Mirica, K. Conductive two-dimensional metal-organic frameworks as multifunctional materials. Chem. Commun. 2018, 54, 7873-7891.

(30) Mähringer, A.; Jakowetz, A. C.; Rotter, J. M.; Bohn, B. J.; Feldmann, J.; Bein, T.; Medina, D.D. oriented Thin Films of Electroactive Triphenylene catecholate-Based 2D Metal-Organic Frameworks. ACS Nano, 2019. 


\section{A highly Crystalline Anthracene-based MOF-74 Series featuring Electrical Conductivity and Luminescence}

Patricia I. Scheurle ${ }^{\ddagger+\nabla}$, Andre Mähringer ${ }^{\ddagger+\nabla}$, Andreas C. Jakowetz ${ }^{\dagger \nabla}$, Pouya Hosseini ${ }^{\infty}$, Alexander F. Richter ${ }^{\nabla}$, , Gunther Wittstock ${ }^{\infty}$, Dana D. Medina ${ }^{\nabla^{*}}$ and Thomas Bein ${ }^{\nabla+^{*}}$

${ }^{\dagger}$ Department of Chemistry, Ludwig-Maximilians-Universität (LMU), Butenandtstr. 5-13 (E), 81377 Munich, Germany.

${ }^{\perp}$ Chair for Photonics and Optoelectronics, Nano-Institute Munich, Department of Physics, Ludwig-Maximilians-Universität (LMU), Königinstr. 10, 80539 Munich, Germany.

${ }^{\nabla}$ Nanosystems Initiative Munich (NIM) and Center for NanoScience (CeNS), Schellingstr. 4, 80799 Munich, Germany.

${ }^{\infty}$ Institute of Chemistry, Carl von Ossietzky Universität Oldenburg, 26111 Oldenburg, Germany.

\section{Table of Contents}

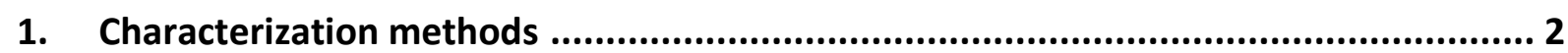

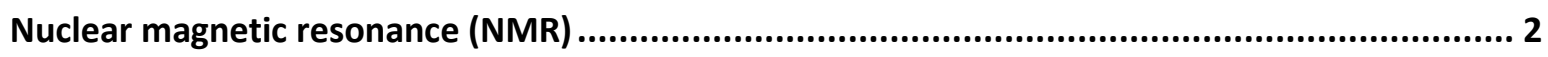

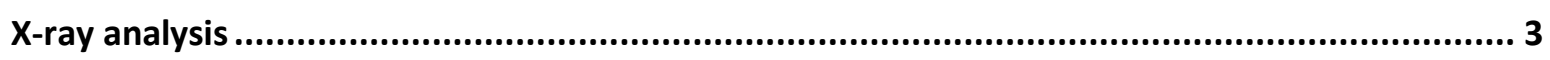

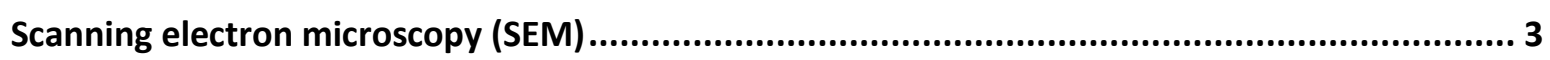

Transmission electron microscopy (TEM) ............................................................................. 3

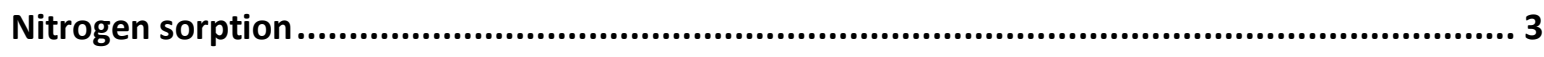

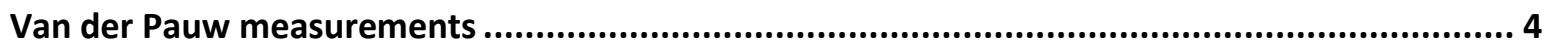

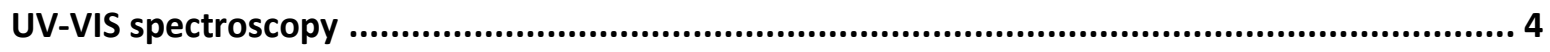

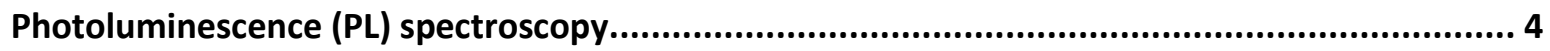

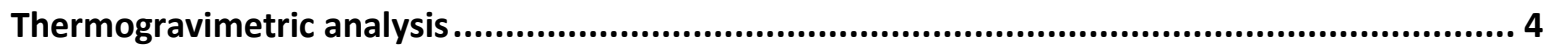

X-ray photoelectron spectroscopy

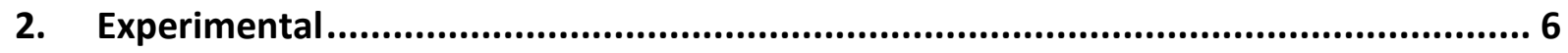

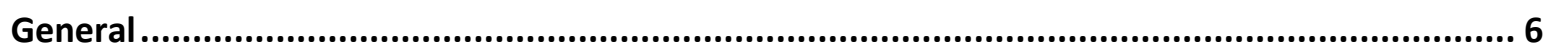

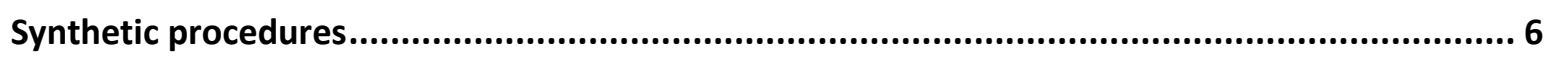

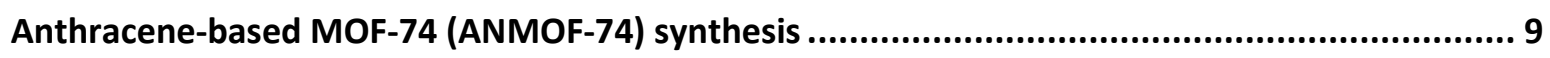

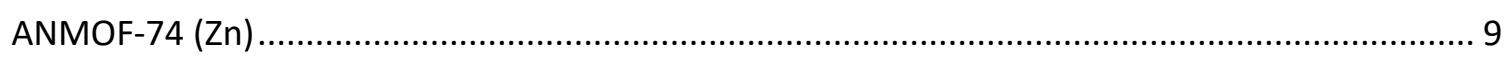


ANMOF-74 (Mg)

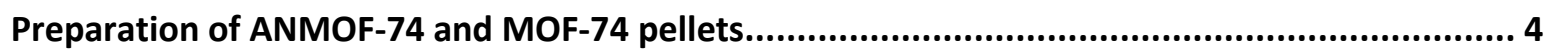

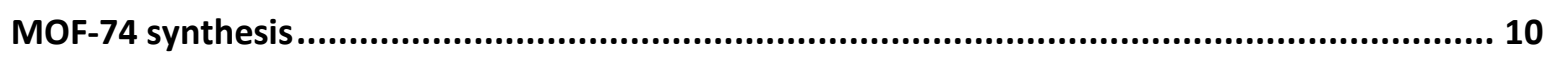

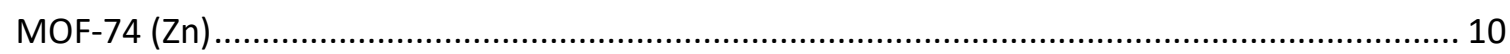

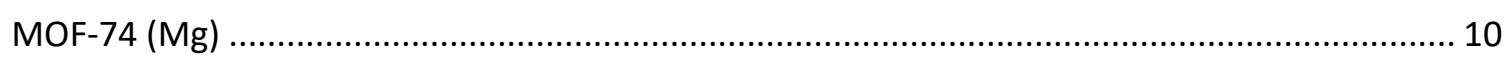

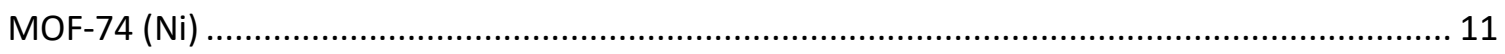

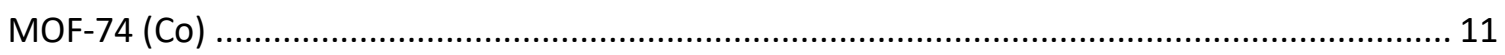

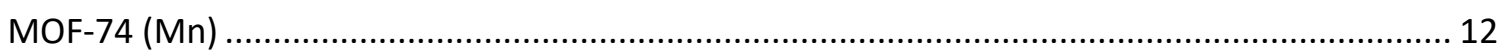

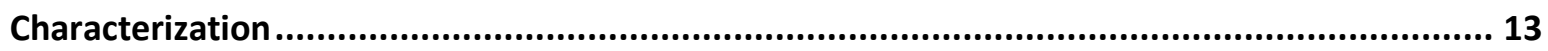

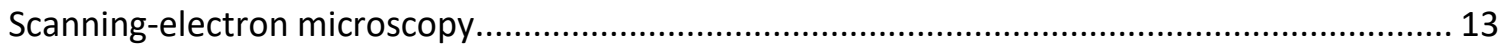

UV-VIS spectroscopy and photoluminescence of the ABHB linker ........................................... 14

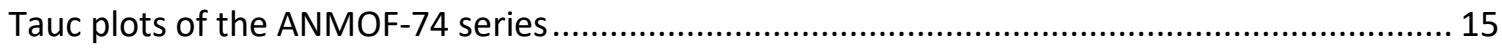

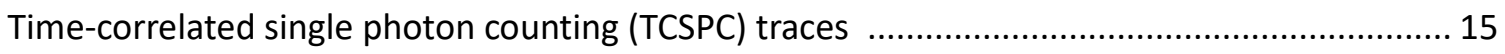

Photoluminescence quantum yield (PLQY) and CIE color space ............................................. 17

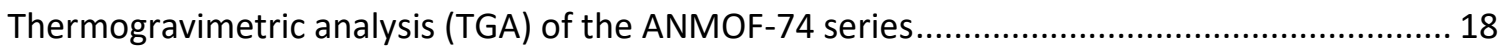

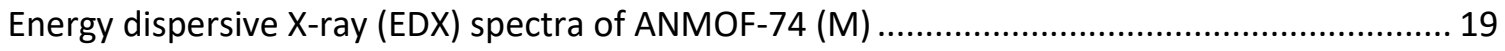

X-ray photoelectron spectroscopy (XPS) of the ANMOF-74 series ............................................ 20

\section{Characterization methods}

\section{Nuclear magnetic resonance (NMR)}

NMR spectra were recorded on Bruker AV400 and AV400 TR spectrometers. The chemical shifts are expressed in parts per million and calibrated using residual (undeuterated) solvent 
peaks as an internal reference ( ${ }^{1} \mathrm{H}-\mathrm{NMR}$ : DMSO- $d_{6}: 2.50 ;{ }^{13} \mathrm{C}-\mathrm{NMR}$ : DMSO- $\left.d_{6}: 39.52\right)$. The data for ${ }^{1} \mathrm{H}$-NMR spectra are written as follows: $s=$ singlet, $d=$ doublet, $t=$ triplet, $q=$ quartet, $\mathrm{m}=$ multiplet.

\section{X-ray analysis}

X-ray diffraction (XRD) analyses were performed on a Bruker D8 diffractometer in BraggBrentano geometry with Ni-filtered $\mathrm{Cu} \mathrm{K}_{\alpha}(\lambda=1.54060 \AA)$ radiation operating at $40 \mathrm{kV}$ and $30 \mathrm{~mA}$ with a position-sensitive detector (LynxEye).

\section{Scanning electron microscopy (SEM)}

SEM images were recorded on a FEI Helios NanoLab G3 UC electron microscope with an acceleration voltage of $2 \mathrm{kV}$ from a field emission gun. For the cross-section analysis substrates were partially cut and broken manually to reveal fresh cross-sections. Prior to SEM analysis the samples were coated with a thin carbon layer by carbon fiber flash evaporation in high vacuum.

\section{Transmission electron microscopy (TEM)}

TEM images were collected on an FEI Titan Themis 60-300 microscope at an acceleration voltage of $300 \mathrm{kV}$. Powder samples were prepared by crushing the particles with a razor blade and subsequently depositing the powder onto a copper grid supporting a thin electron transparent carbon film.

\section{Nitrogen sorption}

Ad- and desorption measurements were performed on an Autosorb 1 (Quantachrome instruments, Florida, USA) with nitrogen of $99.9999 \%$ purity at $77.3 \mathrm{~K}$. The samples were activated (dried) under high vacuum at $120^{\circ} \mathrm{C}$ for at least $12 \mathrm{~h}$. Evaluation of ad-and desorption isotherms was carried out with the AsiQwin v.3.01 (Quantachrome instruments, Florida, USA) software.

For BET calculations, pressure ranges of the nitrogen isotherms were chosen with the help of the BET assistant in the AsiQwin software. In accordance with the ISO recommendations, multipoint BET tags equal to or below the maximum in $V \times\left(1-p / p_{0}\right)$ were chosen. 


\section{Van der Pauw measurements}

Van der Pauw measurements were conducted on a ECOPIA Model HMS 3000 Hall measurement setup. The samples were fixed in an SPCB-1 spring clip board without any additional contacts. Distances between the single probes were adjusted to be $5 \mathrm{~mm}$.

\section{Preparation of ANMOF-74 and MOF-74 pellets}

ANMMOF-74 and MOF-74 pellets with $1 \mathrm{~cm}$ diameter (obtained from several described batches) for electrical conductivity measurements were fabricated with $100 \mathrm{mg}$ of the respective MOF bulk material with a standard Paul-Weber $\mathrm{KBr}$ Press with $45 \mathrm{~kg} / \mathrm{cm}^{2}$ pressure.

\section{UV-VIS spectroscopy}

Photophysical characterization of as-prepared MOF thin films on $2.0 \mathrm{~cm} \times 1.5 \mathrm{~cm}$ quartz substrates was performed in transmission mode with a PERKIN ELMER UV VIS/NIR Lambda 1050 spectrophotometer equipped with a $150 \mathrm{~mm}$ integrating sphere.

\section{Photoluminescence (PL) spectroscopy}

PL measurements were performed on a PicoQuant FluoTime 300 time-correlated single photon counting (TCSPC) setup. Steady-state spectra and time-resolved histograms were acquired using a $378 \mathrm{~nm}$ laser (PicoQuant LDH-P-C-375). Residual laser scattering was removed using a $400 \mathrm{~nm}$ dielectric long pass (Thorlabs FELHO4OO) in the beam path of the emitted light, which was detected under magic angle $\left(54.7^{\circ}\right)$ on a photomultiplying tube (PicoQuant PMA 192).

For measuring the absolute photoluminescence quantum yield and $\mathrm{CIE}-$ values, we used a Horiba FluoroLog 3 in combination with an integrating sphere by Horiba. The sample was prepared as follows: a small amount of powder of the MOF was sandwiched between two glass coverslips and sealed using Parafilm at the edges. A reference structure without sample was prepared as well. By relating the signal area change between sample and reference at the luminescence region and at the Rayleigh peak the absolute radiative quantum yield was calculated.

\section{Thermogravimetric analysis}

Thermogravimetric analysis (TGA) measurements were performed on a Netzsch Jupiter ST 449 C instrument equipped with a Netzsch TASC 414/4 controller. The samples were heated 
from room temperature to $800{ }^{\circ} \mathrm{C}$ under a synthetic air flow $\left(25 \mathrm{~mL} \mathrm{~min}^{-1}\right)$ at a heating rate of $1 \mathrm{~K} \mathrm{~min}^{-1}$.

\section{X-ray photoelectron spectroscopy}

XPS was performed using an ESCALAB 250 Xi instrument (Thermo Fisher, East Grinsted, UK) with monochromatized $\mathrm{Al} \mathrm{K \alpha}(\mathrm{hv}=1486.6 \mathrm{eV})$ radiation focused to a spot of 500 micron diameter at the surface of samples. Spectra were measured with pass energies of $200 \mathrm{eV}$ for survey scans and $10 \mathrm{eV}$ for high-resolution regions. Charging was compensated by use of an internal electron flood gun. Peak fitting was performed by the software Avantage, version 5.9904 (Thermo Fisher) using a Shirley background ("Smart Shirley") and a convolution of Gaussian and Lorentzian functions for each signal component. All spectra were referenced to remaining adventitious carbon at $284.8 \mathrm{eV}$. 


\section{Experimental}

\section{General}

All materials were purchased from Sigma Aldrich, Acros or TCI Europe in the common purities purum, puriss or reagent grade. The materials were used as received without additional purification and handled in air unless otherwise noted.

The water utilized in the synthesis was subjected to a Merck-Milipore Mili-Q purification system prior to use.

\section{Synthetic procedures}

Methyl 2-hydroxy-4-(4,4,5,5-tetramethyl-1,3,2-dioxaborolan-2-yl)benzoate (compound 3) (adapted from Fracaroli, A. M.; Furukawat, H.; Suzuki, M.; Dodd, M.; Okajima, S.; Gandarat, F.; Reimer, J. A.; Yaghi, O. M. Metal-organic Frameworks with Preciseley Designed Interior for Carbon Dioxide Capture in the Presence of Water. J. Am. Chem. Soc. 2014, 136, 8863-8866.)

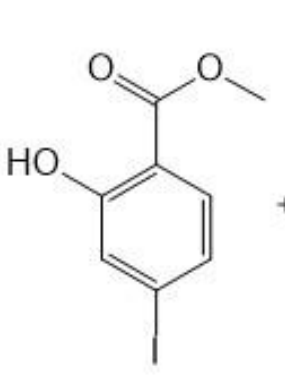

1

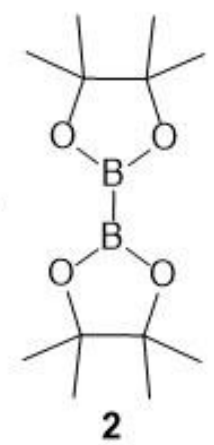

$\mathrm{KOAC}$ $\mathrm{PdCl}_{2}\left(\mathrm{PPh}_{3}\right)_{2}$

1,4-dioxan $130^{\circ} \mathrm{C}, 16 \mathrm{~h}$<smiles>COC(=O)c1ccc(B2OC(C)(C)C(C)(C)O2)cc1O</smiles>

\section{Scheme S1.}

Methyl 4-iodosalicylate (compound 1) $(1.0 \mathrm{~g}, 3.6 \mathrm{mmol})$, bis(pinacolato)diboron (compound 2) $(1.01 \mathrm{~g}, 3.98 \mathrm{mmol}), \mathrm{KOAc}(1.1 \mathrm{~g}, 10.8 \mathrm{mmol})$, and $\mathrm{PdCl}_{2}\left(\mathrm{PPh}_{3}\right)_{2}(50.7 \mathrm{mg}, 0.072 \mathrm{mmol})$ were dissolved in $18 \mathrm{~mL}$ anhydrous 1,4-dioxane in a $100 \mathrm{~mL}$ flask under nitrogen atmosphere. The resulting solution was heated to $130{ }^{\circ} \mathrm{C}$ for $13 \mathrm{~h}$ under stirring. The solution was allowed to cool to room temperature and filtered through celite. It was washed with 1,4-dioxane, toluene and ethyl acetate. The filtrate was concentrated in vacuo. The obtained yellow solid was dissolved in ethyl acetate. The white solid was filtered off. The filtrate was concentrated under vacuum. The obtained yellow needles were recrystallized in methanol $(2 \mathrm{~mL})$, giving colorless crystals with $71 \%$ yield. ${ }^{1} \mathrm{H}$ NMR (400 MHz, DMSO- $\left.d_{6}\right) \delta(p p m): 10.35$ (s, $\left.1 \mathrm{H}\right), 7.75$ (d, $\left.1 \mathrm{H}\right) 7.20$ (s, 1H), $7.17(\mathrm{~d}, 1 \mathrm{H}), 3.87(\mathrm{~s}, 3 \mathrm{H}), 1.26(\mathrm{~s}, 12 \mathrm{H})$. 
Dimethyl 4,4'-(anthracene-9,10-diyl)bis(2-hydroxybenzoate) (compound 5) (adapted from Hirofumi M., Akira S., Yusuke M., Yoshinori I. Polycarboxylic acid containing condensed aromatic ring, crystalline network complex using same, and gas storage material. 2017. WO 2017006638 A1.)<smiles>COC(=O)c1ccc(B2OC(C)(C)C(C)(C)O2)cc1O</smiles>

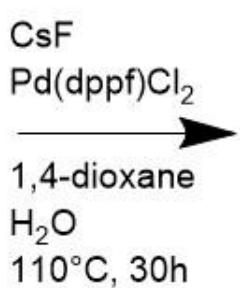<smiles>COC(=O)c1ccc(-c2c3ccccc3c(-c3ccc(C(=O)OC)c(O)c3)c3ccccc23)cc1O</smiles>

\section{Scheme S2.}

Methyl 2-hydroxy-4-(4,4,5,5-tetramethyl-1,3,2-dioxaborolan-2-yl)benzoate (compound 3) (700 mg, $2.5 \mathrm{mmol}$ ), 9,10-dibromoanthracene (compound 4) (369 mg, $1.099 \mathrm{mmol}$ ), CsF (1.0 g, $65.8 \mu \mathrm{mol}), \mathrm{Pd}(\mathrm{dppf}) \mathrm{Cl}_{2}(44.85 \mathrm{mg}, 613 \mu \mathrm{mol})$ were dissolved in $12.8 \mathrm{~mL}$ 1,4-dioxane and 1.8 $\mathrm{mL}$ water in a $100 \mathrm{~mL}$ flask under nitrogen atmosphere. The resulting solution was heated to $110{ }^{\circ} \mathrm{C}$ for $30 \mathrm{~h}$. The solution was allowed to cool to room temperature and $\mathrm{NH}_{4} \mathrm{Cl}(40 \mathrm{~mL})$ and chloroform (40 mL) were added. It was filtered through celite and the residue was washed with chloroform $(400 \mathrm{~mL})$. The filtrate was separated and the aqueous layer was extracted with chloroform. The organic layer was washed with saturated sodium chloride solution $(3 \times 200$ $\mathrm{mL}$ ) and dried over $\mathrm{MgSO}_{4}$. The filtrate was concentrated under vacuum, giving a brown powder with $72 \%$ yield. ${ }^{1} \mathrm{H}$ NMR (400 MHz, DMSO- $\left.d_{6}\right) \delta(\mathrm{ppm}): 8.04(\mathrm{~d}, 2 \mathrm{H}), 7.64-7.59(\mathrm{~m}, 4 \mathrm{H})$, 7.49-7.45 (m, 4H), $7.09(t, 2 H), 7.06-7.03(d d d, 2 H), 3.99(s, 6 H)$. 
4,4'-(anthracene-9,10-diyl)bis(2-hydroxybenzoic acid) (compound 6) ABHB linker, (adapted from Hirofumi M., Akira S., Yusuke M., Yoshinori I. (2017). WO 2017006638 A1.)
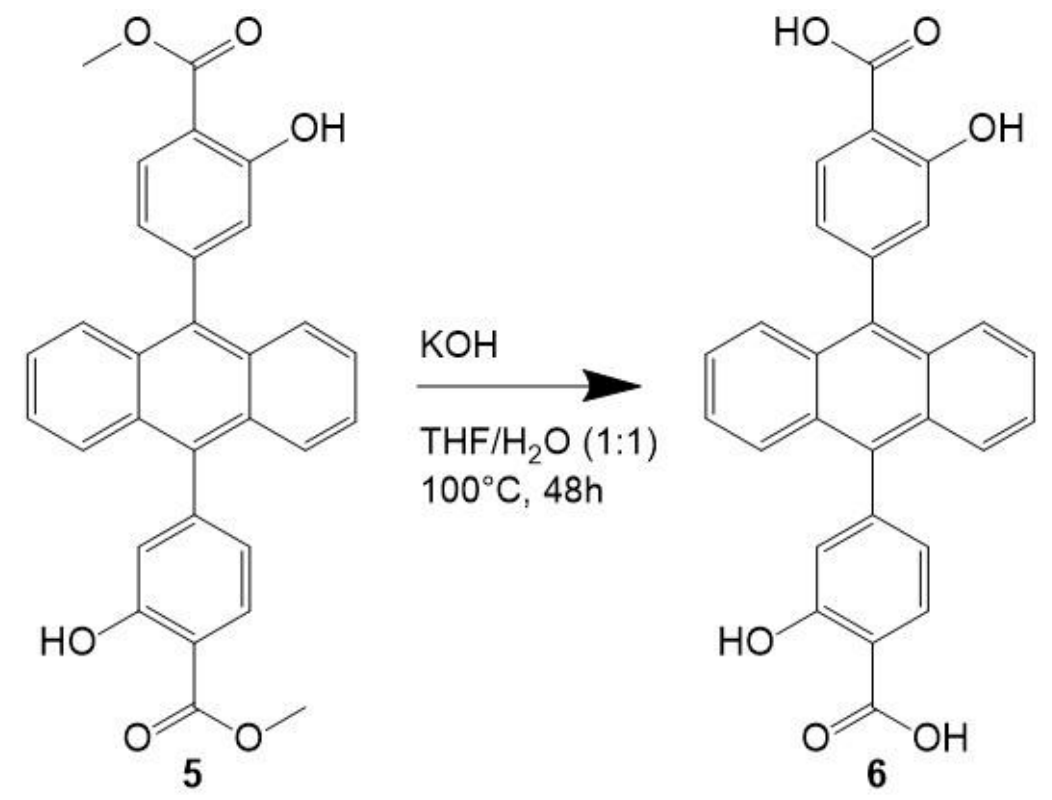

\section{Scheme S3.}

Dimethyl 4,4'-(anthracene-9,10-diyl)bis(2-hydroxybenzoate) (compound 5) (300 mg, $0.63 \mathrm{mmol})$, potassium hydroxide $(\mathrm{KOH})(500 \mathrm{mg}, 8.9 \mathrm{mmol})$ and a mixture of $\mathrm{THF} / \mathrm{H}_{2} \mathrm{O}(1: 1)$ $(30 \mathrm{~mL})$ were heated in a $100 \mathrm{~mL}$ flask under nitrogen atmosphere at $100{ }^{\circ} \mathrm{C}$ for $48 \mathrm{~h}$. The resulting solution was allowed to cool to room temperature and poured into $\mathrm{HCl}(200 \mathrm{~mL})$. The solid was filtered off, washed with acetic acid $(30 \mathrm{~mL})$, water $(500 \mathrm{~mL})$ and $\mathrm{MeOH}(30 \mathrm{~mL})$ and dried under vacuum, giving a beige powder with $70 \%$ yield. ${ }^{1} \mathrm{H}$ NMR (400 MHz, DMSO- $d_{6}$ ) $\delta(\mathrm{ppm}): 8.03(\mathrm{~d}, 2 \mathrm{H}), 7.64-7.59(\mathrm{~m}, 4 \mathrm{H}), 7.48-7.43(\mathrm{~m}, 4 \mathrm{H}), 7.03(\mathrm{~s}, 2 \mathrm{H}), 7.02-6.97(\mathrm{~m}, 2 \mathrm{H}), 3.99$ (s, 6H). ${ }^{13} \mathrm{C}$ NMR (400 MHz, DMSO-d $\left.d_{6}\right) \delta$ (ppm): 171.73 (2C), 161.07 (2C), 145.75 (2C), 135.59 (2C), 130.60 (3C), $128.51(3 C), 126.13$ (6C), 125.92 (3C), $122.11(4 C)$. 


\section{Anthracene-based MOF-74 (ANMOF-74) synthesis}

\section{ANMOF-74(Zn)}

4,4'-(anthracene-9,10-diyl)bis(2-hydroxybenzoic acid) (10.00 mg, $0.022 \mathrm{mmol}), \mathrm{Zn}\left(\mathrm{NO}_{3}\right)_{2}$. $4 \mathrm{H}_{2} \mathrm{O}$ (22.07 $\left.\mathrm{mg}, 0.084 \mathrm{mmol}\right)$ and benzoic acid $(2.7 \mathrm{mg}, 0.022 \mathrm{mmol})$ were suspended in DMF $(1.5 \mathrm{~mL})$. The resulting mixture was sonicated until complete dissolution of the educts was achieved and it was kept in a culture tube at $120^{\circ} \mathrm{C}$ for 2 days. The resulting precipitate was washed with $\mathrm{DMF}(2 \times 10 \mathrm{~mL})$ and $\mathrm{MeOH}(1 \times 10 \mathrm{~mL})$ and activated at $120^{\circ} \mathrm{C}$ to remove the less volatile solvent to obtain a brownish crystalline powder. The PXRD pattern of the synthesized MOF matches the simulated ANMOF-74 PXRD pattern.

\section{ANMOF-74(Mg)}

4,4'-(anthracene-9,10-diyl)bis(2-hydroxybenzoic acid) (10.00 mg, $0.022 \mathrm{mmol}$ ) and $\mathrm{Mg}\left(\mathrm{NO}_{3}\right)_{2}$ - $6 \mathrm{H}_{2} \mathrm{O}$ (21.60 mg, $\left.0.084 \mathrm{mmol}\right)$ were suspended in DMF (1.3 mL) and $\mathrm{MeOH}(0.2 \mathrm{~mL})$. The resulting mixture was sonicated until complete dissolution of the educts was achieved and it was kept in a culture tube at $120^{\circ} \mathrm{C}$ for 2 days. The resulting precipitate was washed with DMF $(2 \times 10 \mathrm{~mL})$ and $\mathrm{MeOH}(1 \times 10 \mathrm{~mL})$ and activated at $120^{\circ} \mathrm{C}$ to remove the less volatile solvent to obtain a bright beige crystalline powder. The PXRD pattern of the synthesized MOF matches the simulated ANMOF-74 PXRD pattern.

\section{ANMOF-74(Ni)}

4,4'-(anthracene-9,10-diyl)bis(2-hydroxybenzoic acid) (10.00 mg, $0.022 \mathrm{mmol}), \mathrm{NiCl}_{2}$ (10.80 $\mathrm{mg}, 0.084 \mathrm{mmol}$ ) and benzoic acid $(2.7 \mathrm{mg}, 0.022 \mathrm{mmol}$ ) were suspended in benzyl alcohol $(0.7 \mathrm{~mL})$ and $\mathrm{EtOH}(0.7 \mathrm{~mL})$ The resulting mixture was sonicated until complete dissolution of the educts was achieved and it was kept in a culture tube at $120{ }^{\circ} \mathrm{C}$ for 2 days. The resulting precipitate was washed with DMF $(2 \times 10 \mathrm{~mL})$ and $\mathrm{MeOH}(1 \times 10 \mathrm{~mL})$ and activated at $120^{\circ} \mathrm{C}$ to remove the less volatile solvent to obtain a green crystalline powder. The PXRD pattern of the synthesized MOF matches the simulated ANMOF-74 PXRD pattern.

\section{ANMOF-74(Co)}

4,4'-(anthracene-9,10-diyl)bis(2-hydroxybenzoic acid) (10.00 mg, $0.022 \mathrm{mmol}), \mathrm{Co}\left(\mathrm{NO}_{3}\right)_{2}$. $6 \mathrm{H}_{2} \mathrm{O}(24.30 \mathrm{mg}, 0.084 \mathrm{mmol})$ and benzoic acid $(2.7 \mathrm{mg}, 0.022 \mathrm{mmol})$ were suspended in DMF $(1.1 \mathrm{~mL}), \mathrm{EtOH}(0.3 \mathrm{~mL})$ and $\mathrm{H}_{2} \mathrm{O}(1 \mathrm{~mL})$. The resulting mixture was sonicated until complete 
dissolution of the educts was achieved and it was kept in a culture tube at $120^{\circ} \mathrm{C}$ for 2 days. The resulting precipitate was washed with DMF $(2 \times 10 \mathrm{~mL})$ and $\mathrm{MeOH}(1 \times 10 \mathrm{~mL})$ and activated at $120^{\circ} \mathrm{C}$ to remove the less volatile solvent to obtain a pink crystalline powder. The PXRD pattern of the synthesized MOF matches the simulated ANMOF-74 PXRD pattern.

\section{ANMOF-74(Mn)}

4,4'-(anthracene-9,10-diyl)bis(2-hydroxybenzoic acid) (10.00 mg, $0.022 \mathrm{mmol}$ ) and $\mathrm{MnCl}_{2}$. $4 \mathrm{H}_{2} \mathrm{O}$ (22.60 mg, $\left.0.084 \mathrm{mmol}\right)$ were suspended in DMF $(1.5 \mathrm{~mL})$. The resulting mixture was sonicated until complete dissolution of the educts was achieved and it was kept in a culture tube at $120^{\circ} \mathrm{C}$ for 2 days. The resulting precipitate was washed with DMF $(2 \times 10 \mathrm{~mL})$ and $\mathrm{MeOH}(1 \times 10 \mathrm{~mL})$ and activated at $120^{\circ} \mathrm{C}$ to remove the less volatile solvent to obtain a light beige crystalline powder. The PXRD pattern of the synthesized MOF matches the simulated ANMOF-74 PXRD pattern.

\section{MOF-74 synthesis}

\section{$\operatorname{MOF-74(Zn)}$}

The synthesis was adapted from: Rosi, N. L.; Kim, J.; Eddaoudi, M.; Chen, B.; O’Keeffe, M.; Yaghi, O. M. Rod Packings and metal-Organic Frameworks Constructed from Rod-Shaped Secondary Building Units. J. Am. Chem. Soc. 2005, 127, 1504-1518.

2,5-dihydroxy-1,4-benzenedicarboxylic acid (0.019 g, $0.096 \mathrm{mmol})$ and zinc nitrate tetrahydrate, $\mathrm{Zn}\left(\mathrm{NO}_{3}\right)_{2} \cdot 4 \mathrm{H}_{2} \mathrm{O}(0.053 \mathrm{mg}, 0.203 \mathrm{mmol})$ were dissolved in DMF $(2 \mathrm{~mL}), 2$ propanol $(0.1 \mathrm{~mL})$ and water $(0.1 \mathrm{~mL})$. The resulting mixture was sonicated until complete dissolution of the educts was achieved and it was kept in a culture tube at $105^{\circ} \mathrm{C}$ for 1 day. The resulting precipitate was washed with DMF $(2 \times 10 \mathrm{~mL})$ and ethanol $(1 \times 10 \mathrm{~mL})$ and dried under reduced pressure.

\section{MOF-74(Mg)}

The synthesis was adapted from: Caskey, S. R.; Wong-Foy, A. G.; Matzger, A. J. Dramatic Tuning of Carbon Dioxide Uptake via Metal Substitution in a Coordination Polymer with Cylindrical Pores. J. Am. Chem. Soc. 2008, 130, 18070-18071. 
2,5-dihydroxy-1,4-benzenedicarboxylic acid (0.111 g, $0.559 \mathrm{mmol})$ and magnesia nitrate hexahydrate, $\mathrm{Mg}\left(\mathrm{NO}_{3}\right)_{2} \cdot 6 \mathrm{H}_{2} \mathrm{O}(0.475 \mathrm{mg}, 1.85 \mathrm{mmol})$ were dissolved in a 15:1:1 mixture of DMF-ethanol-water $(50 \mathrm{~mL})$. The resulting mixture was sonicated until complete dissolution of the educts was achieved and it was kept in a Teflon sealed $100 \mathrm{~mL}$ Schott Duran glass bottle at $125^{\circ} \mathrm{C}$ for 1 day. The resulting precipitate was washed with DMF $(2 \times 10 \mathrm{~mL})$ and methanol $(1 \times 10 \mathrm{~mL})$ and dried under reduced pressure.

\section{MOF-74(Ni)}

The synthesis was adapted from: Caskey, S. R.; Wong-Foy, A. G.; Matzger, A. J. Dramatic Tuning of Carbon Dioxide Uptake via Metal Substitution in a Coordination Polymer with Cylindrical Pores. J. Am. Chem. Soc. 2008, 130, 18070-18071.

2,5-dihydroxy-1,4-benzenedicarboxylic acid (0.478 g, $2.41 \mathrm{mmol})$ and nickel nitrate hexahydrate, $\mathrm{Ni}\left(\mathrm{NO}_{3}\right)_{2} \cdot 6 \mathrm{H}_{2} \mathrm{O}(2.378 \mathrm{mg}, 8.178 \mathrm{mmol})$ were dissolved in a 1:1:1 mixture of DMF-ethanol-water $(200 \mathrm{~mL})$. The resulting mixture was sonicated until complete dissolution of the educts was achieved and it was kept in three Teflon sealed $100 \mathrm{~mL}$ Schott Duran glass bottles at $100^{\circ} \mathrm{C}$ for 1 day. The resulting precipitate was washed with DMF ( $\left.2 \times 10 \mathrm{~mL}\right)$ and methanol $(1 \times 10 \mathrm{~mL})$ and dried under reduced pressure.

\section{MOF-74(Co)}

The synthesis was adapted from: Caskey, S. R.; Wong-Foy, A. G.; Matzger, A. J. Dramatic Tuning of Carbon Dioxide Uptake via Metal Substitution in a Coordination Polymer with Cylindrical Pores. J. Am. Chem. Soc. 2008, 130, 18070-18071.

2,5-dihydroxy-1,4-benzenedicarboxylic acid (0.482 g, $2.43 \mathrm{mmol})$ and cobalt nitrate hexahydrate, $\mathrm{Co}\left(\mathrm{NO}_{3}\right)_{2} \cdot 6 \mathrm{H}_{2} \mathrm{O}(2.377 \mathrm{~g}, 8.67 \mathrm{mmol})$ were dissolved in a 1:1:1 mixture of DMFethanol-water $(200 \mathrm{~mL})$. The resulting mixture was sonicated until complete dissolution of the educts was achieved and it was kept in three Teflon sealed $100 \mathrm{~mL}$ Schott Duran glass bottles at $100^{\circ} \mathrm{C}$ for 1 day. The resulting precipitate was washed with DMF $(2 \times 10 \mathrm{~mL})$ and methanol $(1 \times 10 \mathrm{~mL})$ and dried under reduced pressure. 


\section{MOF-74(Mn)}

The synthesis was adapted from: Cozzolino, A. F.; Brozek, A. K., Palmer, R. D.; Yano, J.; Li, Minyuan; Dinca, M. Ligand Redox Non-Innocence in the Stoichiometric Oxidation of $\mathrm{Mn}_{2}(2,5-$ dioxidoterephtalate) (Mn-MOF-74). J. Am. Chem. Soc. 2014, 136, 3334-3337.

2,5-dihydroxy-1,4-benzenedicarboxylic acid (0.27 g, $1.3 \mathrm{mmol}$ ) and $\mathrm{MnCl}_{2} \cdot 4 \mathrm{H}_{2} \mathrm{O}(1.07 \mathrm{~g}$, $5.41 \mathrm{mmol}$ ) were dissolved in a 15:1 mixture of DMF-ethanol (32 $\mathrm{mL}$ ) under argon atmosphere. The resulting mixture was sonicated until complete dissolution of the educts was achieved and it was kept in a Teflon sealed $100 \mathrm{~mL}$ Schott Duran glass bottle at $135^{\circ} \mathrm{C}$ for 3 day. The resulting precipitate was washed with DMF $(2 \times 10 \mathrm{~mL})$ and methanol $(1 \times 10 \mathrm{~mL})$ and dried under reduced pressure. 


\section{Characterization}

\section{Powder X-ray diffraction}
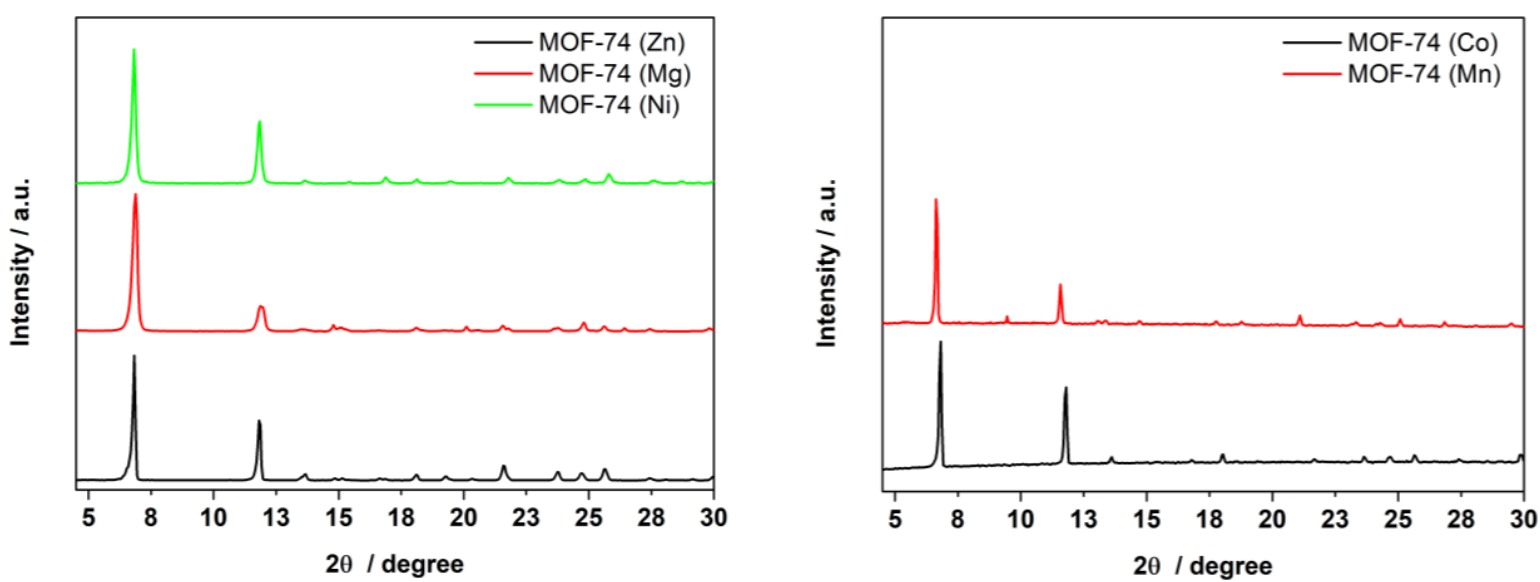

Figure S1: PXRD patterns of the MOF-74(M) bulk materials.

\section{Scanning-electron microscopy}
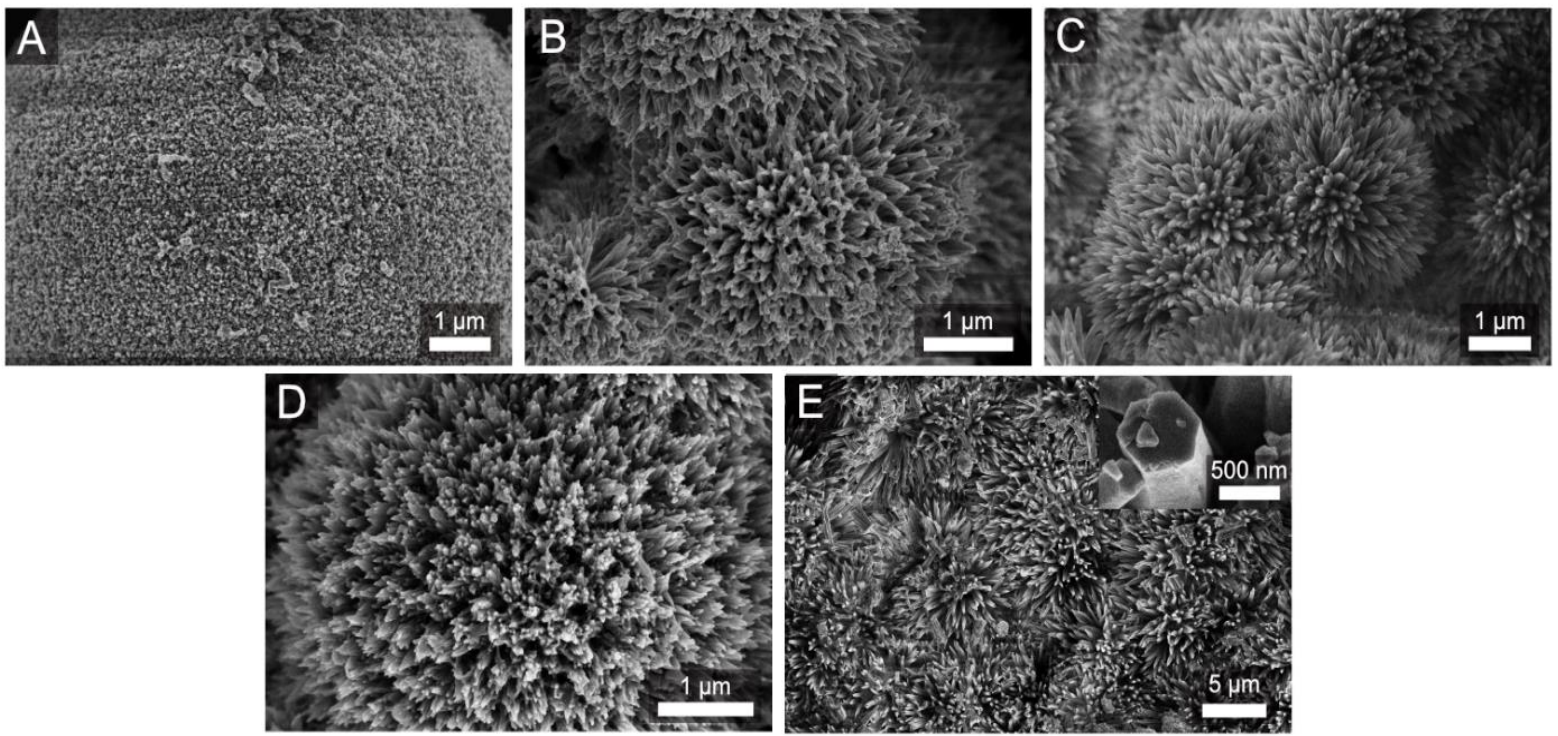

Figure S2: SEM images of the ANMOF-74 bulk materials (A) ANMOF-74(Zn), (B) ANMOF74(Mg), (C) ANMOF-74(Ni), (D) ANMOF-74(Co), (E) ANMOF-74(Mn) and a close-up of a hexagonally shaped monolith depicted as inset. 


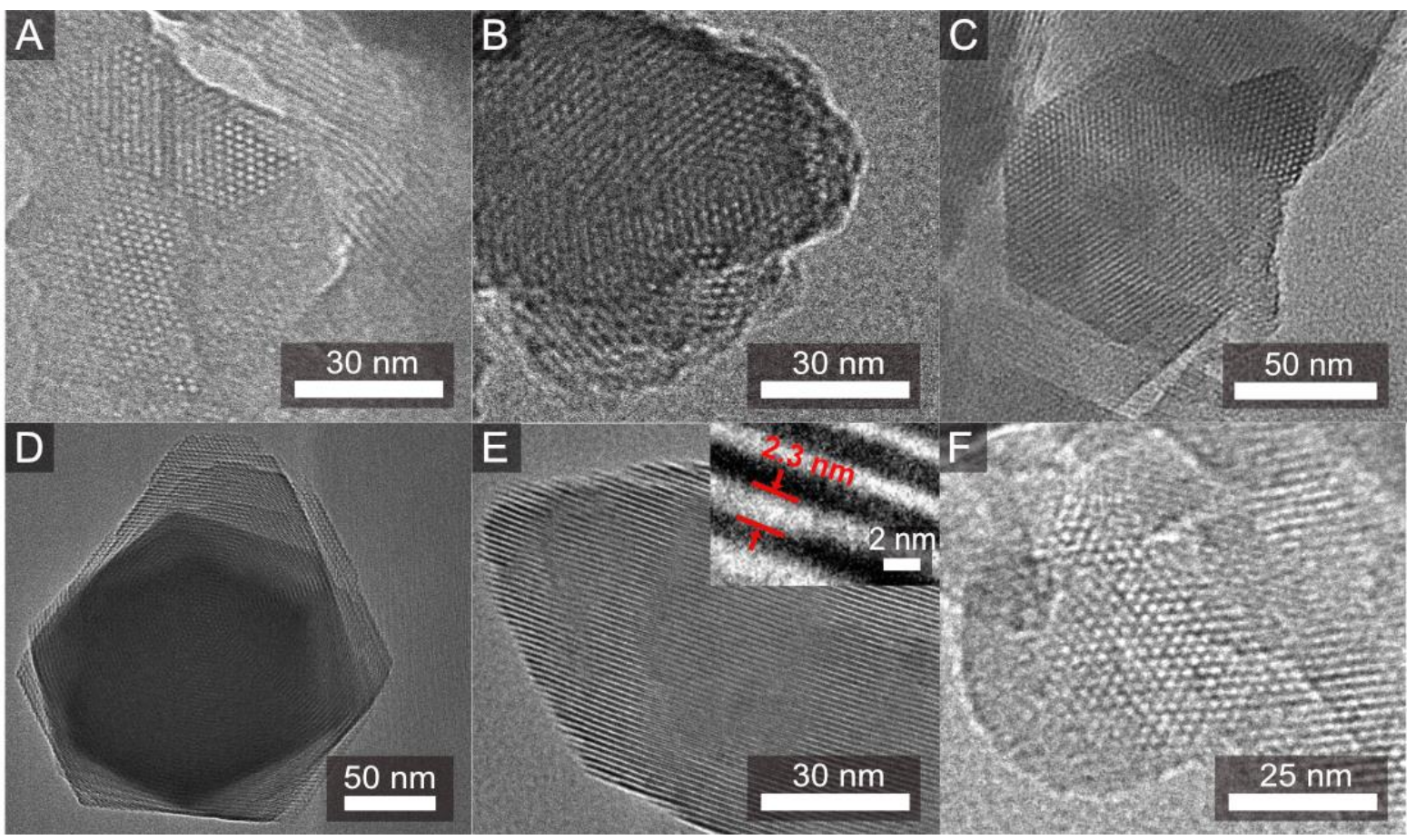

Figure S3: High resolution TEM images of the ANMOF-74(M) series in [001] orientation showing (A) the $\mathrm{Zn-}$, (B) Mg-, (C) Ni-, (D) Co-, (F) Mn-ANMOF-74 materials, respectively. (E) High resolution TEM image of ANMOF-74(Co) in side view showing a close-up of the lattice planes as inset.

\section{UV-VIS spectroscopy and photoluminescence of the ABHB linker}
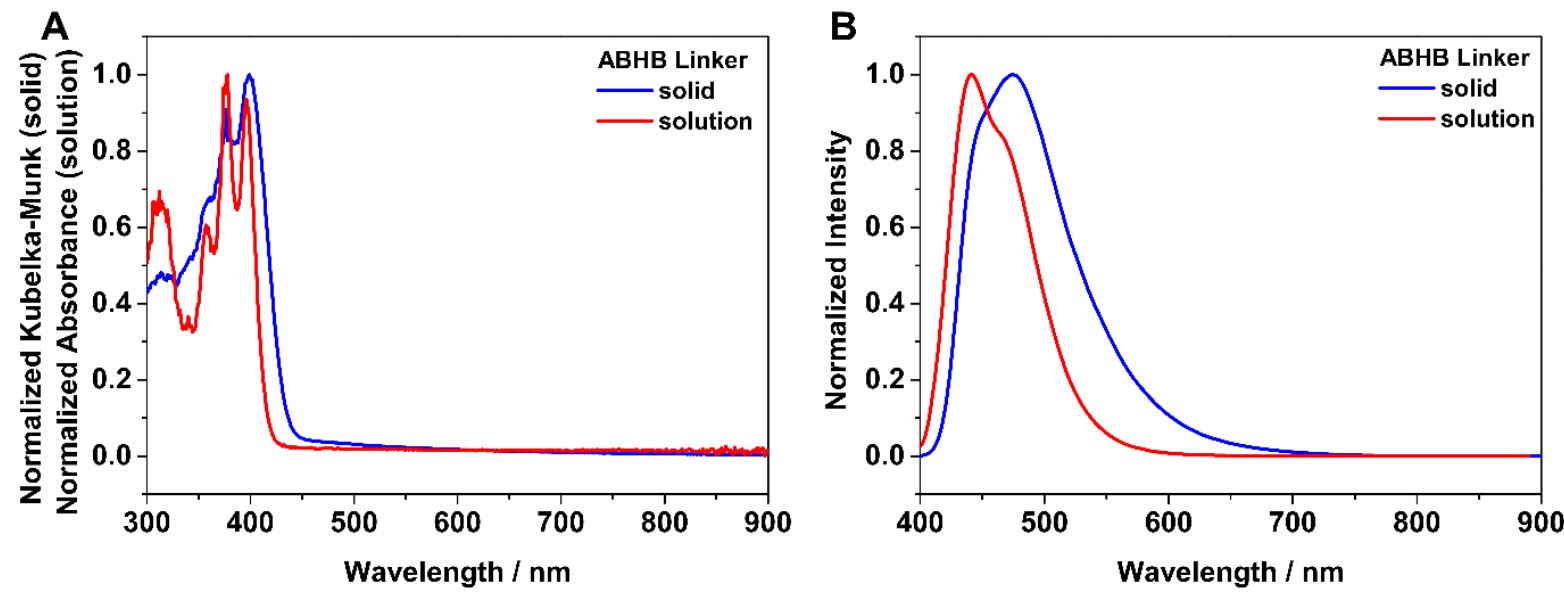

Figure S4: (A) Comparison of the absorption and diffuse reflectance spectra of the ABHB linker in $\mathrm{CHCl}_{3}$ solution (red) and as solid dispersed in $\mathrm{BaSO}_{4}$ (blue). (B) PL spectra of the ABHB linker measured with pulsed $378 \mathrm{~nm}$ excitation in $\mathrm{CHCl}_{3}$ solution (red) and as solid (blue). 
Tauc plots of the ANMOF-74 series
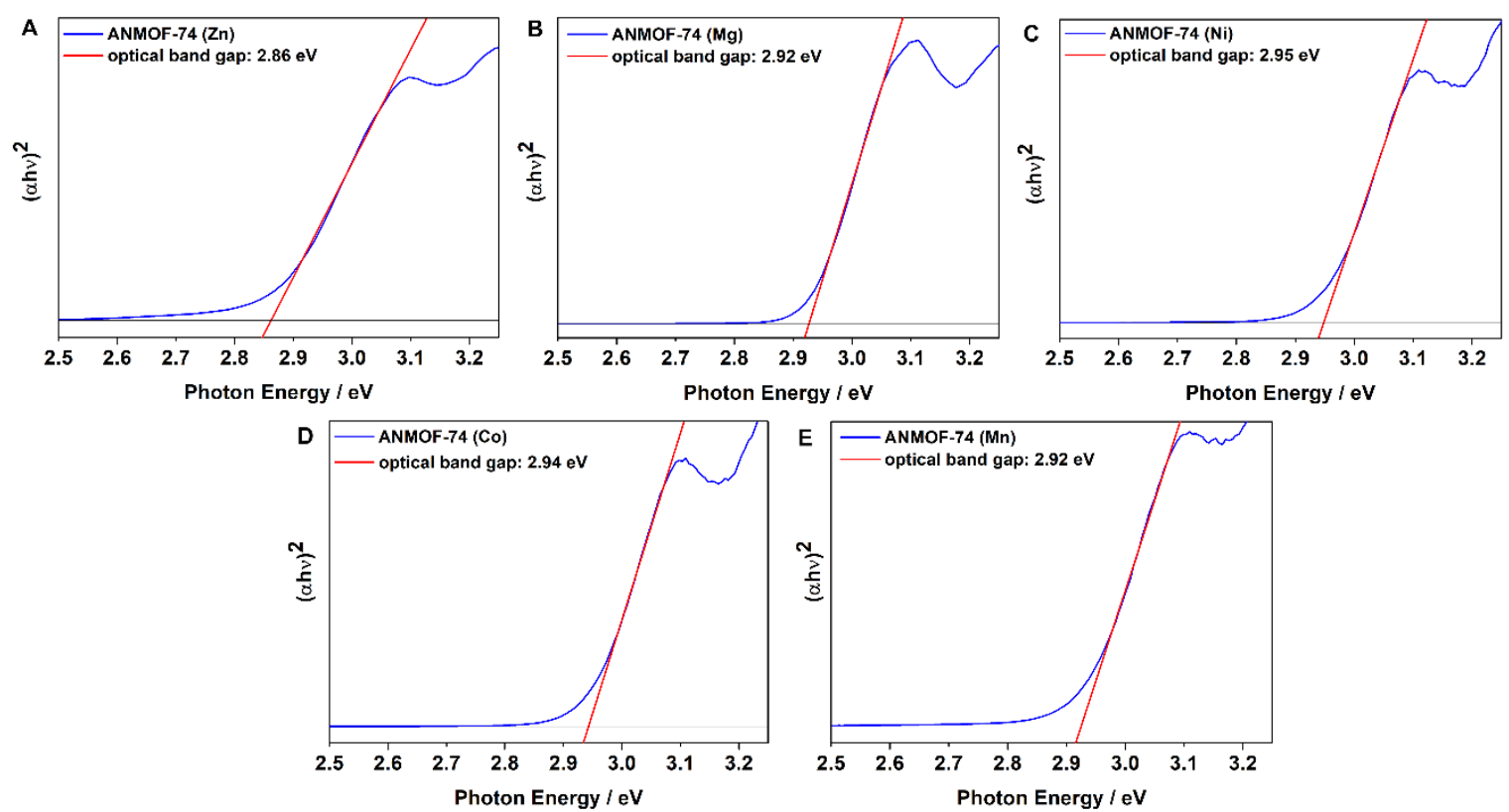

Figure S5: Tauc plots of ANMOF-74(Zn) (A), ANMOF-7(Mg) (B), ANMOF-74 (Ni) (C), ANMOF74(Co) (D) and ANMOF-74(Mn) (E), suggesting the presence of direct band gaps.

Time-correlated single photon counting (TCSPC) traces

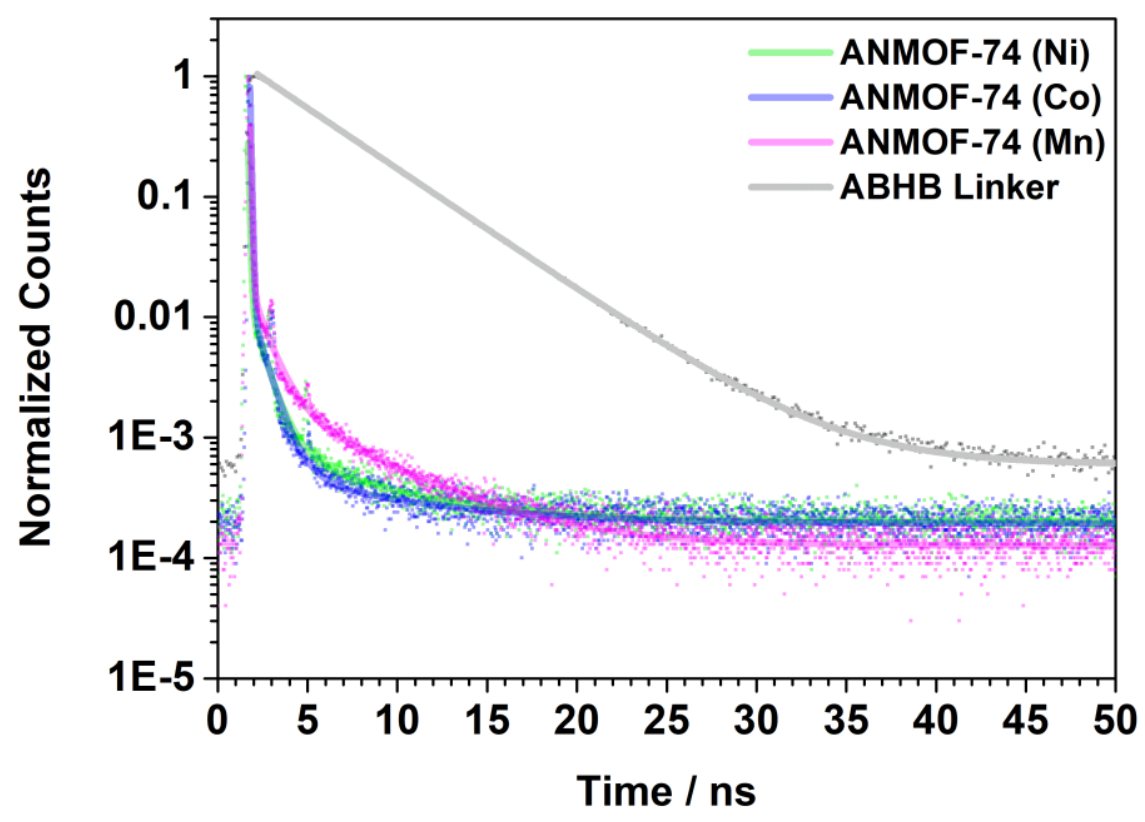

Figure S6: The time-correlated single photon counting (TCSPC) traces corresponding to the PL spectra of ANMOF-74(Ni), ANMOF-74(Co) and ANMOF-74(Mn) as solids and of the ABHB linker 
in solution which were recorded at the respective emission maximum of each material. The solid lines represent the exponential fits.

Table S1: The PL lifetimes $(\tau)$ of the ANMOF-74(M) series and of the ABHB linker, obtained from triexponential fits (see Figure S5 and 6) the TCSPC traces of the MOFs and from a monoexpontial fit of the TCSPC trace of the ABHB linker, as well as the error and the fractional intensities in percentages, which are the fractions of emitted photons corresponding to the respective lifetimes. We note that the stated error is the fitting error and that it does not necessarily reflect the actual resolution of the setup of around $100 \mathrm{ps}$.

\begin{tabular}{|c|c|c|c|c|}
\hline sample & & $\tau / n s$ & error / ns & $\begin{array}{l}\text { fractional } \\
\text { intensity / \% }\end{array}$ \\
\hline \multirow[t]{3}{*}{ ANMOF-74 (Zn) } & $\tau_{1}$ & 0.162 & \pm 0.012 & 22.9 \\
\hline & $\tau_{2}$ & 0.79 & \pm 0.03 & 59.5 \\
\hline & $\tau_{3}$ & 3.4 & \pm 0.2 & 17.6 \\
\hline \multirow[t]{3}{*}{ ANMOF-74 (Mg) } & $\tau_{1}$ & 0.169 & \pm 0.006 & 28.7 \\
\hline & $\tau_{2}$ & 1.03 & \pm 0.02 & 50.0 \\
\hline & $\tau_{3}$ & 4.90 & \pm 0.12 & 21.3 \\
\hline \multirow[t]{3}{*}{ ANMOF-74 (Ni) } & $\tau_{1}$ & 0.065 & \pm 0.004 & 55.3 \\
\hline & $\tau_{2}$ & 0.80 & \pm 0.06 & 31.9 \\
\hline & $\tau_{3}$ & 5.6 & \pm 1.0 & 12.8 \\
\hline \multirow[t]{3}{*}{ ANMOF-74 (Co) } & $\tau_{1}$ & 0.064 & \pm 0.002 & 80.5 \\
\hline & $\tau_{2}$ & 0.79 & \pm 0.06 & 15.5 \\
\hline & $\tau_{3}$ & 7 & \pm 2 & 4.0 \\
\hline \multirow[t]{3}{*}{ ANMOF-74 (Mn) } & $\tau_{1}$ & 0.065 & \pm 0.003 & 48.3 \\
\hline & $\tau_{2}$ & 0.89 & \pm 0.06 & 28.8 \\
\hline & $\tau_{3}$ & 5.1 & \pm 0.4 & 22.9 \\
\hline $\begin{array}{l}\text { Anthracene } \\
\text { (Monomer) }\end{array}$ & $\tau$ & 4.32 & \pm 0.011 & 100 \\
\hline
\end{tabular}


Photoluminescence quantum yield (PLQY) and CIE color space

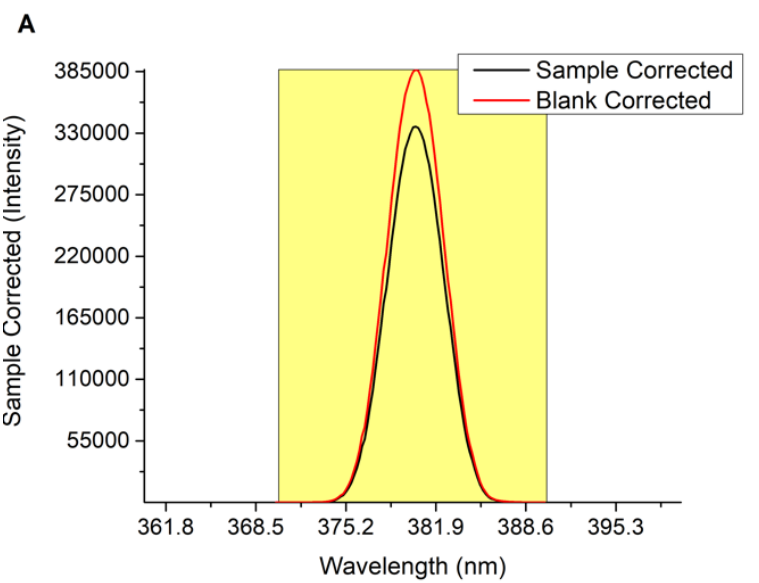

B

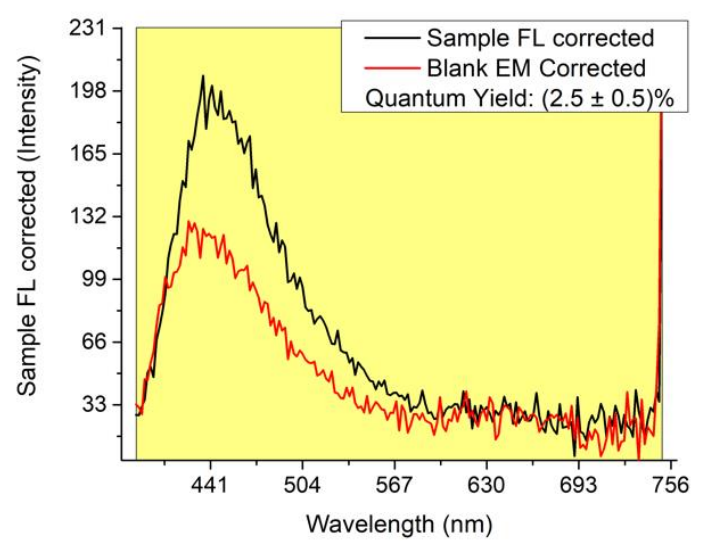

Figure S7: Photoluminescence quantum yield (PLQY) measurement of ANMOF-74(Mg). Rayleigh peak (A) and emission (B) data with integration boundaries.
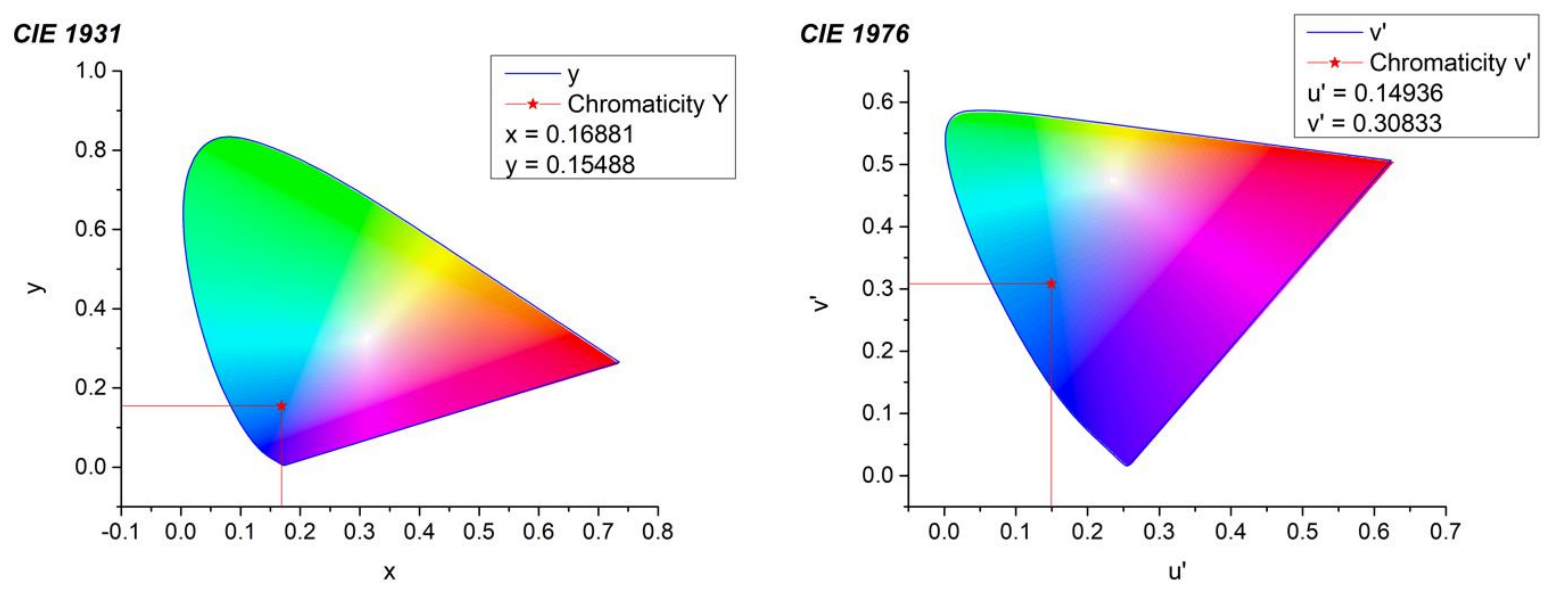

Figure S8: CIE 1931 and CIE 1976 color spaces with the ANMOF-74(Mg) emission and the respective coordinates.

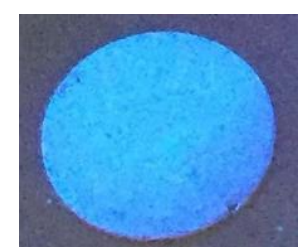

Figure S9: Photo image of a ANMOF-74(Mg) pellet during irradiation with a $365 \mathrm{~nm}$ wavelength. 
Thermogravimetric analysis (TGA) of the ANMOF-74 series
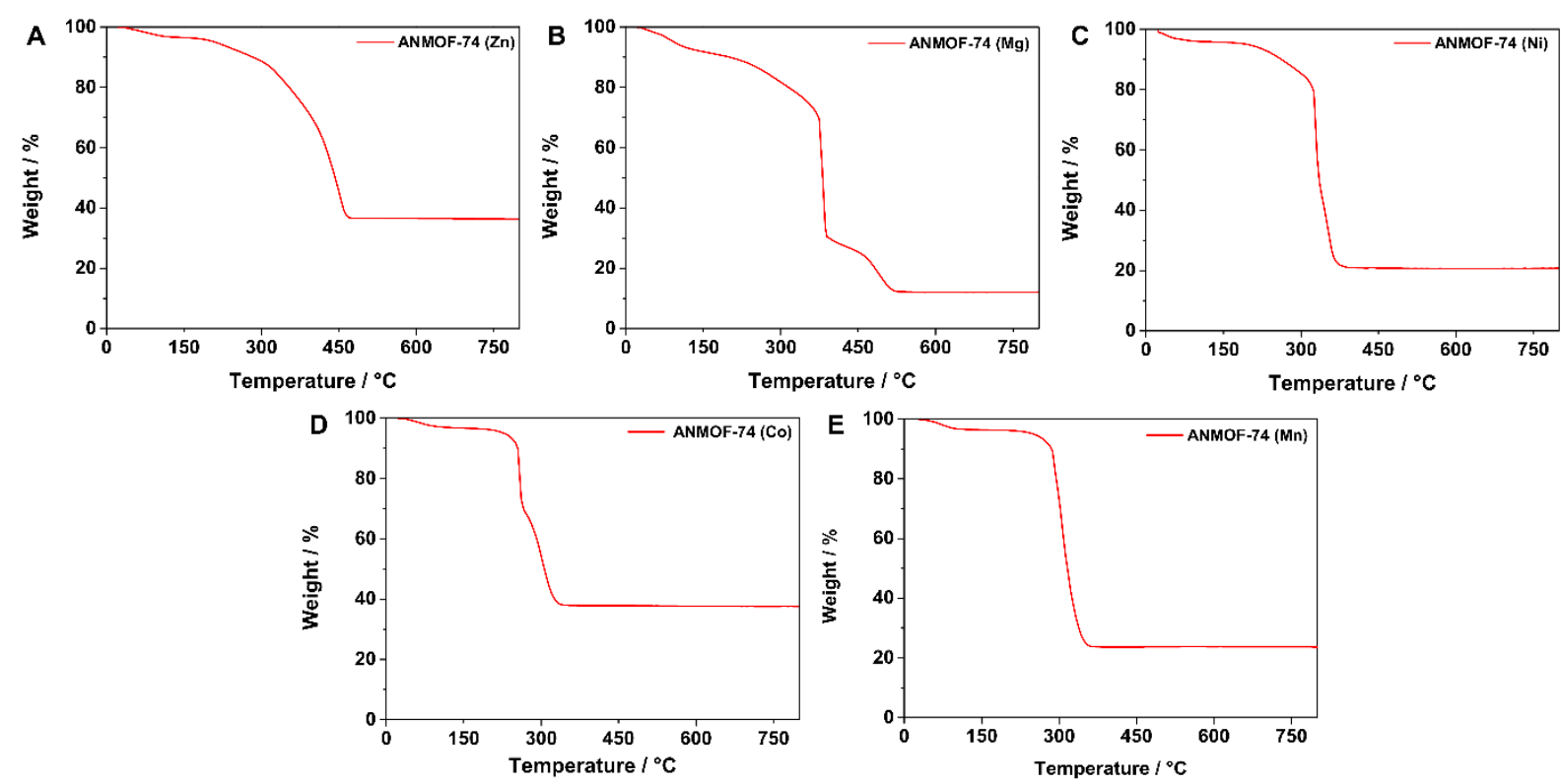

Figure S10: Thermogravimetric Analysis (TGA) of (A) ANMOF-74(Zn), (B) ANMOF-74(Mg), (C), ANMOF-74(Ni), (D) ANMOF-74(Co) and (E) ANMOF-74(Mn) measured as bulk materials (20 mg). 

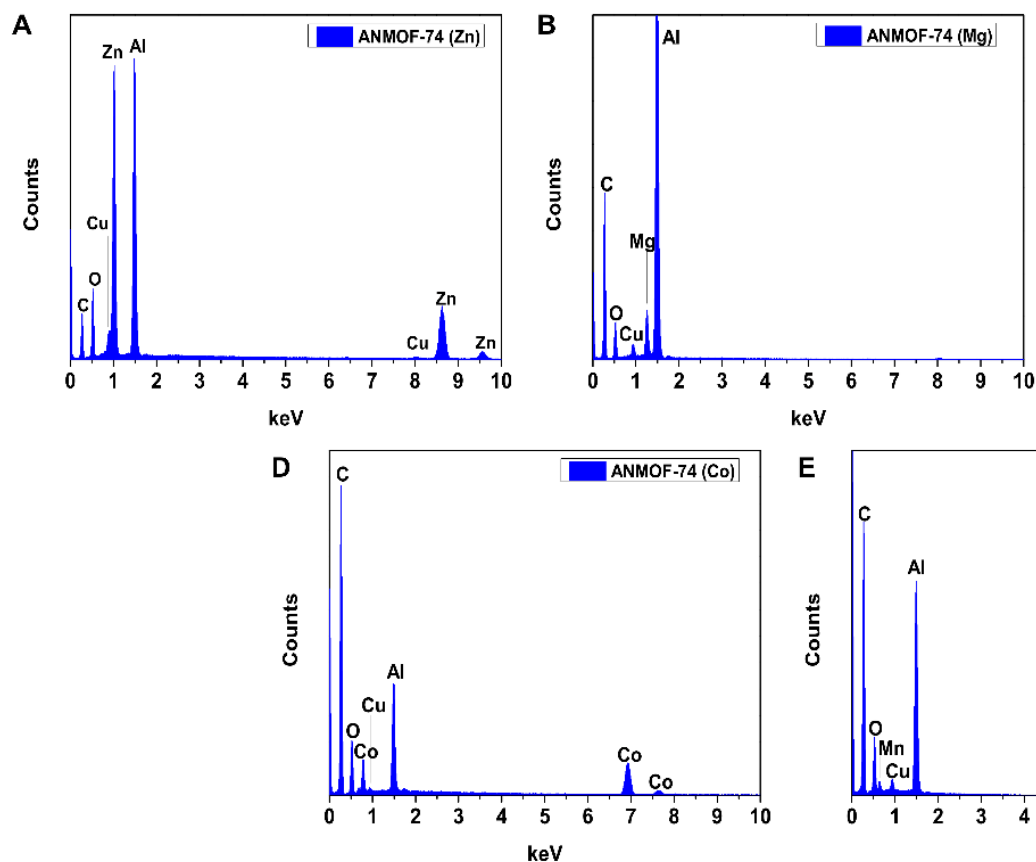
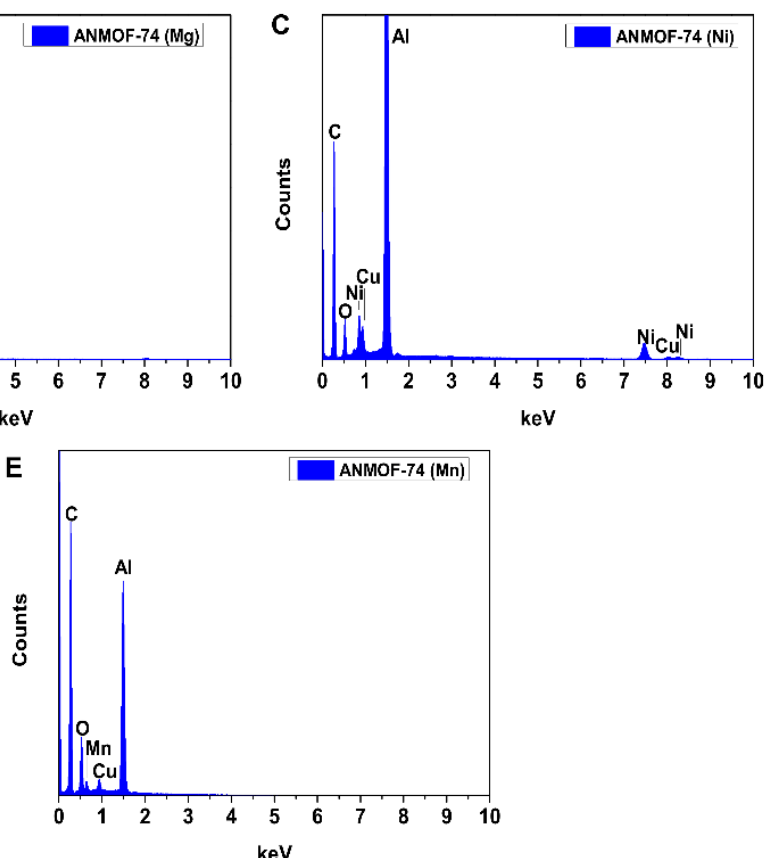

Figure S11: Energy dispersive X-ray (EDX) spectra of (A) ANMOF-74(Zn), (B) ANMOF-74(Mg), (C), ANMOF-74(Ni), (D) ANMOF-74(Co) and (E) ANMOF-74(Mn), measured as bulk materials on an SEM sample holder.

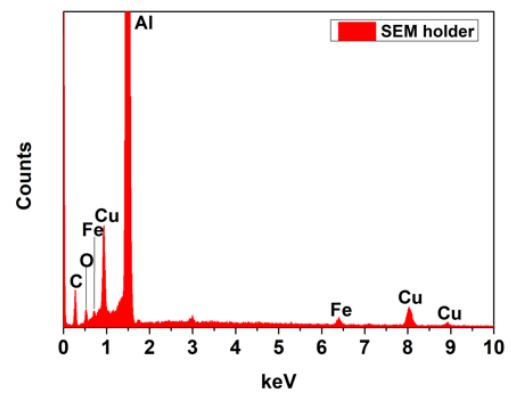

Figure S12: Energy dispersive X-ray (EDX) spectrum of the SEM sample holder. 
X-ray photoelectron spectroscopy (XPS) of the ANMOF-74 series
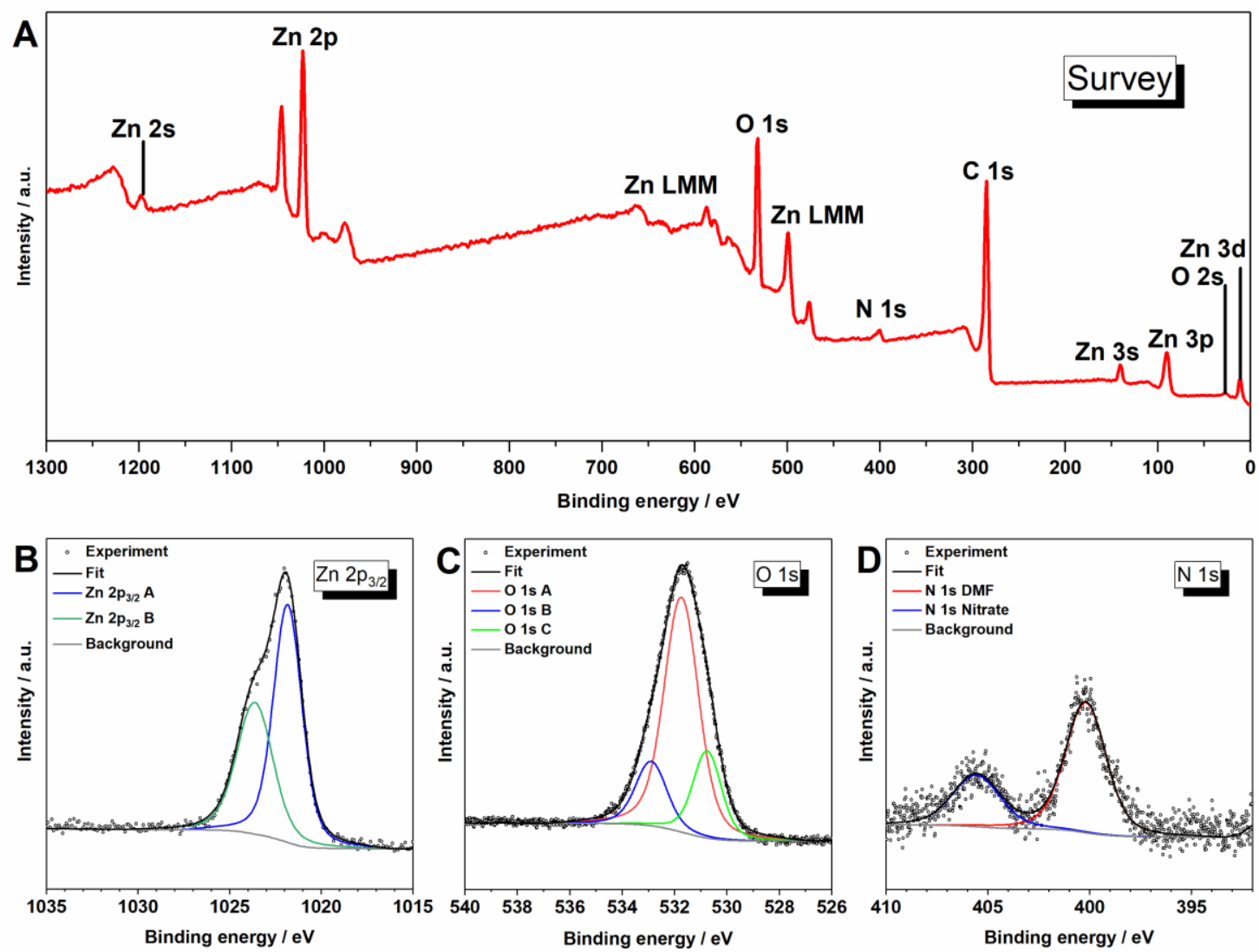

Figure S13: XPS spectra of ANMOF-74(Zn) powder: (A) survey scan, (B) Zn 2p spectrum, (C) $\mathrm{O}$ 1s spectrum and (D) N 1s spectrum. 

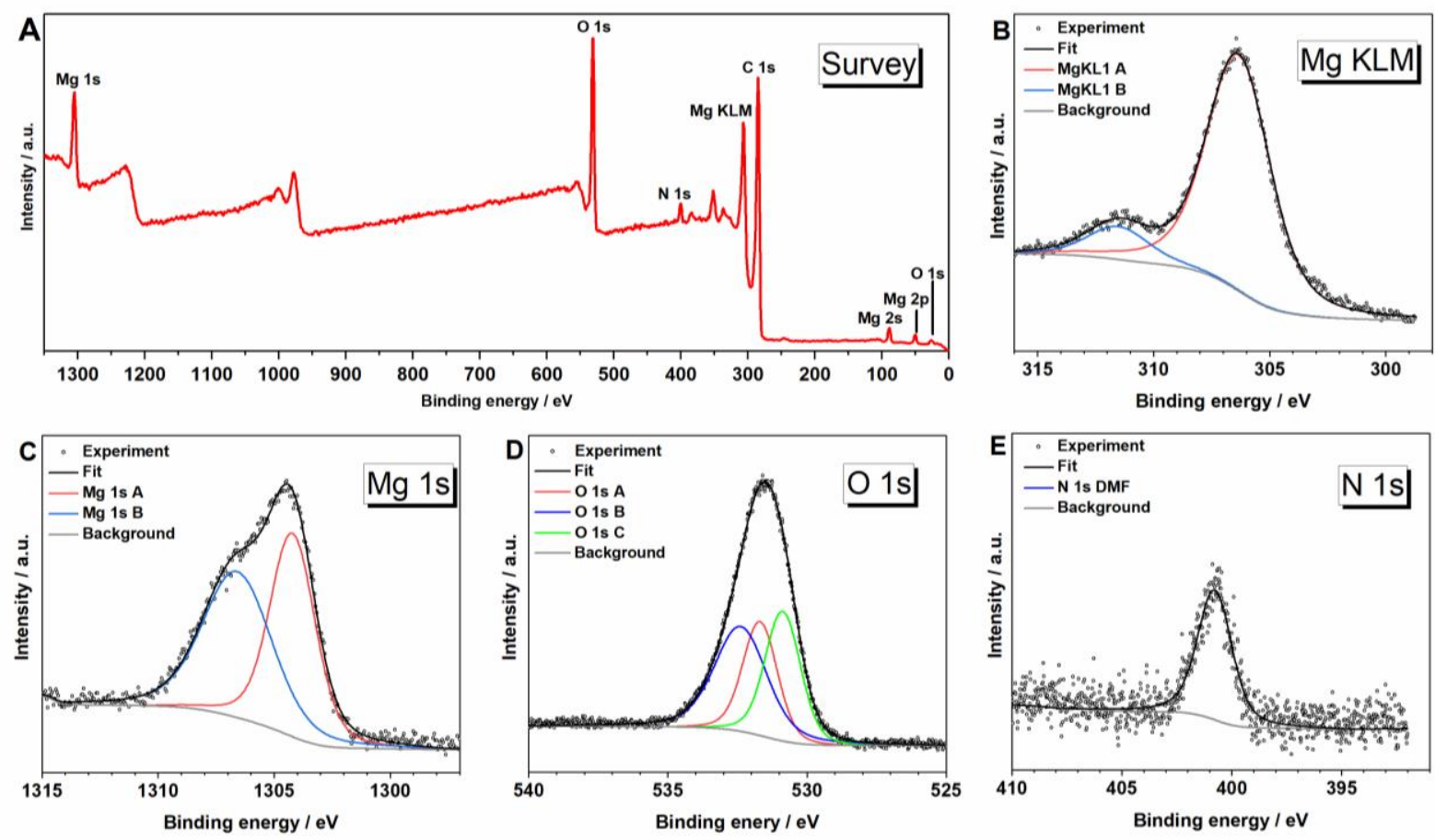

Figure S14: XPS spectra of ANMOF-74(Mg) powder: (A) survey scan, (B) Mg KLM spectrum, (C) Mg 1s spectrum, (D) $O$ 1s spectrum and (E) N 1s spectrum. 

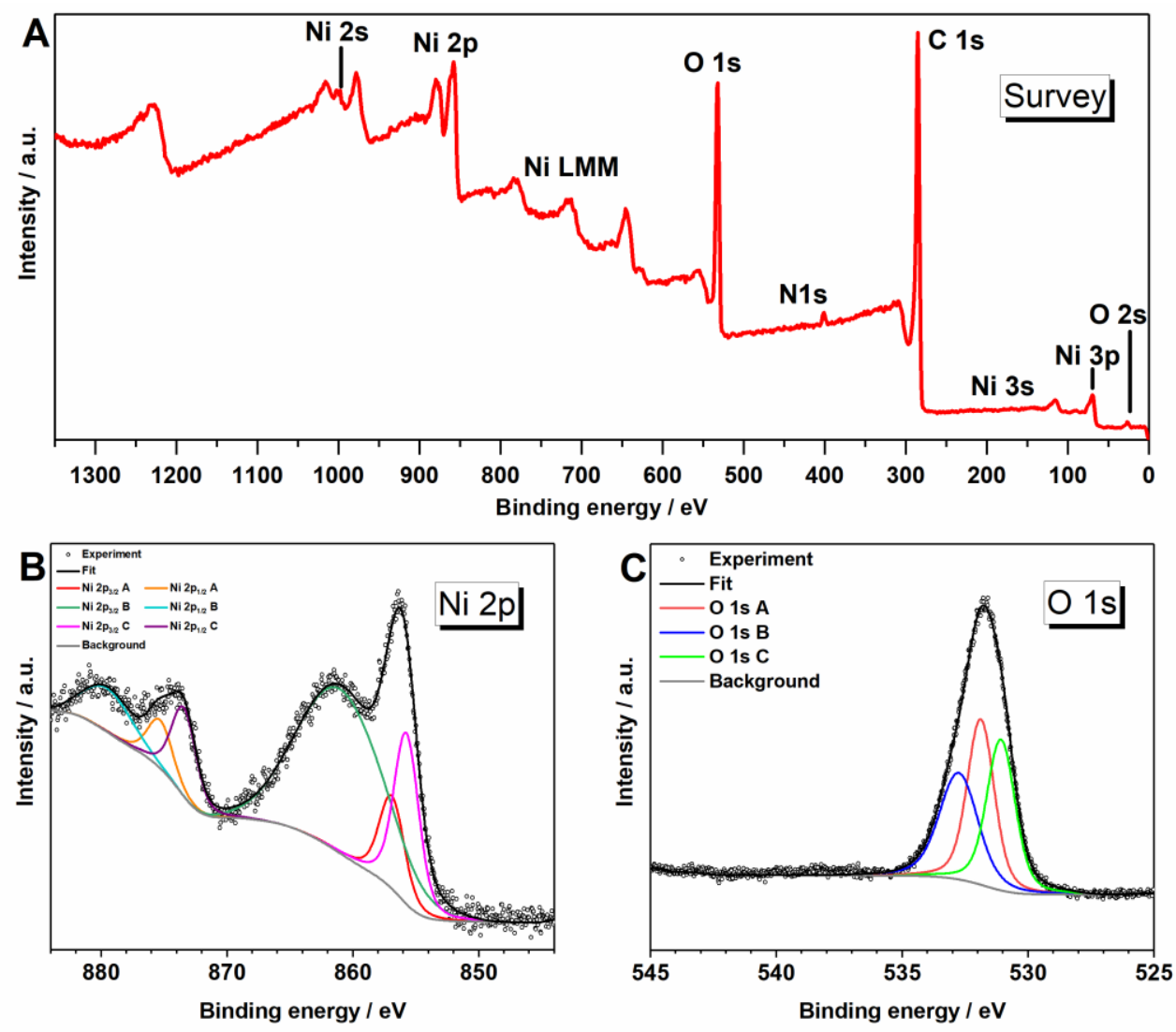

Figure S15: XPS spectra of ANMOF-74(Ni) powder: (A) survey scan, (B) Ni 2p spectrum and (C) O 1s spectrum. 

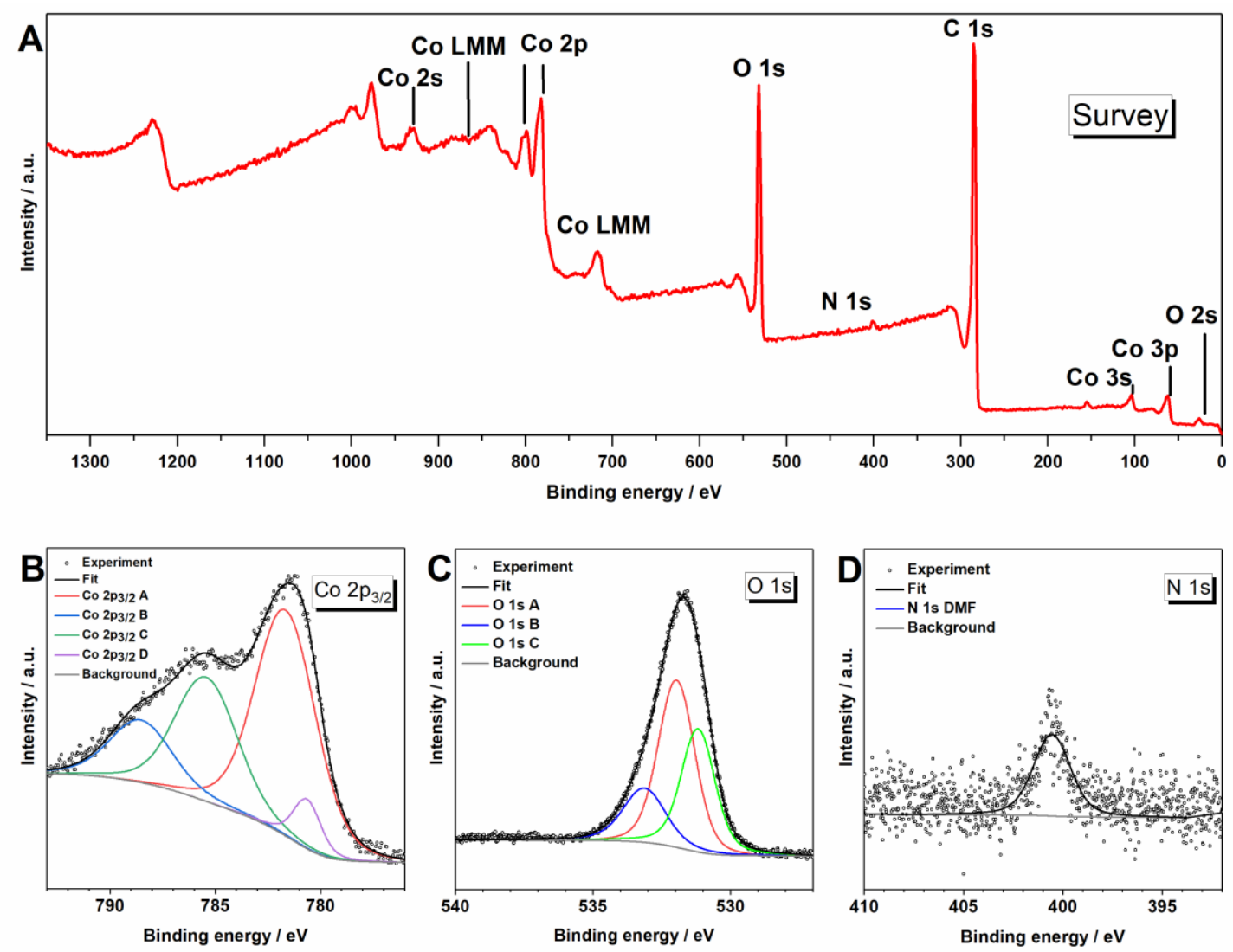

Figure S16: XPS spectra of ANMOF-74(Co) powder: (A) survey scan, (B) Co $2 p$ spectrum, (C) $\mathrm{O}$ 1s spectrum and (D) N 1s spectrum. 

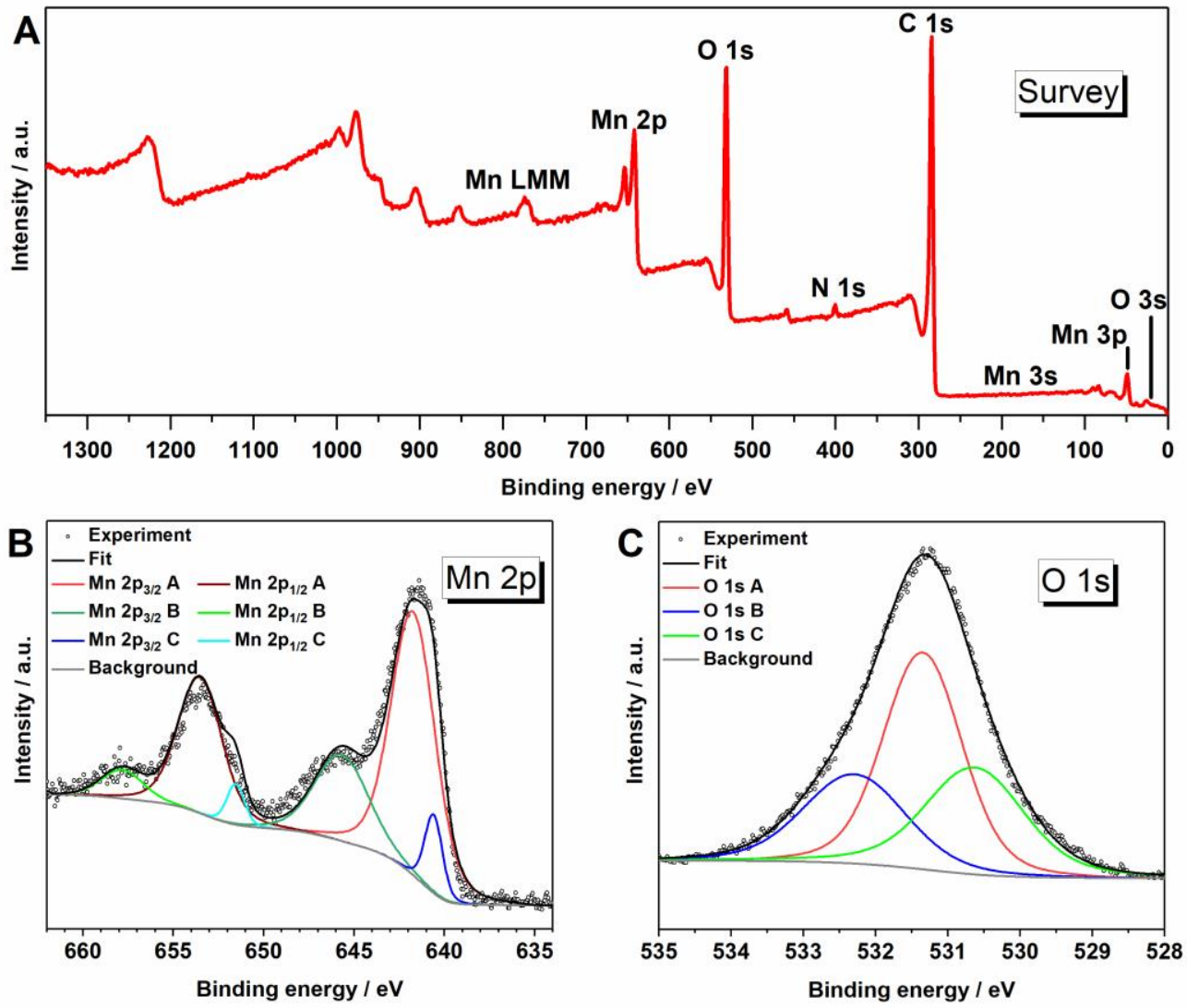

Figure S17: XPS spectra of ANMOF-74(Mn) powder: (A) survey scan, (B) Mn 2p spectrum and

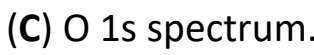


